NASA Technical Memorandum 4728 NASA-TM-4728

19960014633

\title{
A Dynamic Response Model for Pressure Sensors in Continuum and High Knudsen Number Flows with Large Temperature Gradients
}

Stephen A. Whitmore, Brian J. Petersen, and David D. Scott

January 1996
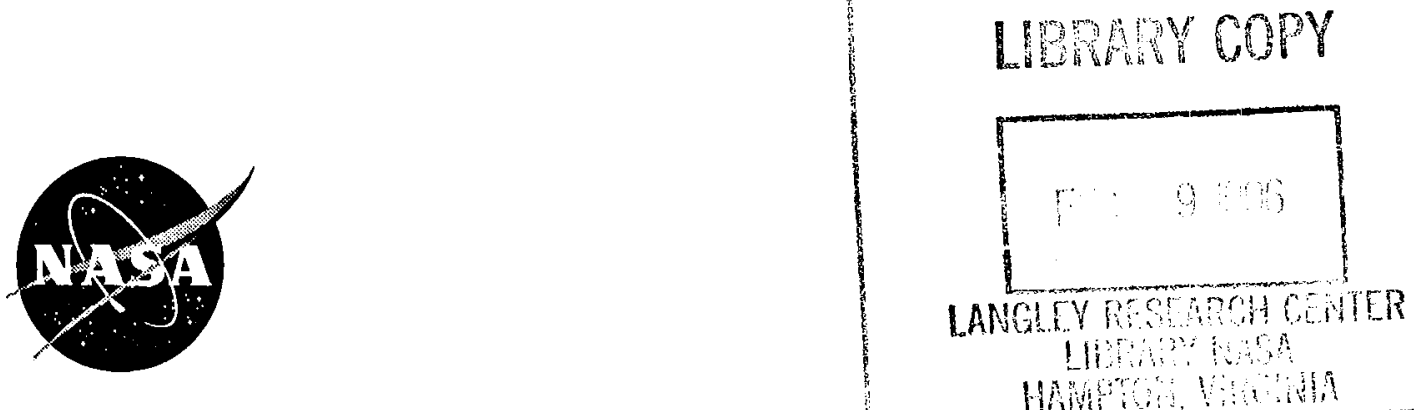



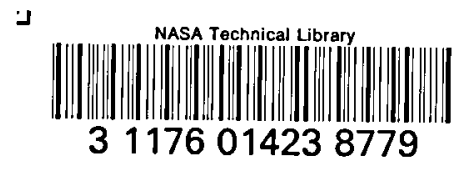

NASA Technical Memorandum 4728

\section{A Dynamic Response Model for Pressure Sensors in Continuum and High Knudsen Number Flows with Large Temperature Gradients}

Stephen A. Whitmore

Dryden Flight Research Center

Edwards, California

Brian J. Petersen

UCLA School of Engineering and Applied Sciences

Los Angeles, California

David D. Scott

Lawrence Livermore National Laboratories

Livermore, California

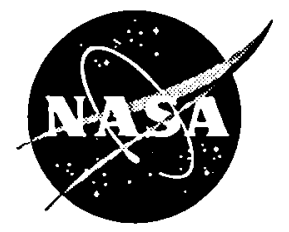

National Aeronautics and

Space Administration

Office of Management

Scientific and Technical Information Program 



\title{
A DYNAMIC RESPONSE MODEL FOR PRESSURE SENSORS IN CONTINUUM AND HIGH KNUDSEN NUMBER FLOWS WITH LARGE TEMPERATURE GRADIENTS
}

\author{
Stephen A. Whitmore* \\ NASA Dryden Flight Research Center \\ Edwards, California 93523 \\ Brian J. Petersen ${ }^{\dagger}$ \\ UCLA School of Engineering and Applied Science \\ Los Angeles, California 90024 \\ David D. Scott ${ }^{\dagger \dagger}$ \\ Lawrence Livermore National Laboratories \\ Livermore, California 91283
}

\begin{abstract}
$\underline{\text { Abstract }}$
This paper develops a dynamic model for pressure sensors in continuum and rarefied flows with longitudinal temperature gradients. The model was developed from the unsteady Navier-Stokes momentum, energy, and continuity equations and was linearized using small perturbations. The energy equation was decoupled from momentum and continuity assuming a polytropic flow process. Rarefied flow conditions were accounted for using a slip flow boundary condition at the tubing wall. The equations were radially averaged and solved assuming gas properties remain constant along a small tubing element. This fundamental solution was used as a building block for arbitrary geometries where fluid properties may also vary longitudinally in the tube. The problem was solved recursively starting at the transducer and working upstream in the tube. Dynamic frequency response tests were performed for continuum flow conditions in the presence of temperature gradients. These tests validated the recursive formulation of the model. Model steady-state

\footnotetext{
* Vehicle Dynamics Group Leader, Aerodynamics Branch, Member, AIAA.

+ Graduate Student, Student Member, AIAA.

t† Engineer.

Copyright $(1) 1996$ by the American Institute of Aeronautics and
} Astronautics, Inc. No copyright is asserted in the United States under Title 17, U.S. Code. The U.S. Government has a royalty-free license to exercise all rights under the copyright claimed herein for Governmental purposes. All other rights are reserved by the copyright owner.
\end{abstract}

behavior was analyzed using the final value theorem. Tests were performed for rarefied flow conditions and compared to the model steady-state response to evaluate the regime of applicability. Model comparisons were excellent for Knudsen numbers up to 0.6. Beyond this point, molecular affects caused model analyses to become inaccurate.

\section{Nomenclature}

\begin{tabular}{|c|c|}
\hline A & temperature profile label, $T_{\max } \sim$ ambient \\
\hline$A_{c}$ & tube cross-section area, in. \\
\hline ac & alternating current \\
\hline $\mathrm{A} / \mathrm{D}$ & analog-to-digital conversion \\
\hline $\mathrm{A}_{\omega}$ & constant of integration, frequency variable \\
\hline B & temperature profile label, $\mathrm{T}_{\max } \sim 350^{\circ} \mathrm{F}$ \\
\hline $\mathrm{B}_{\omega}$ & constant of integration, frequency variable \\
\hline $\mathrm{C}$ & temperature profile label, $\mathrm{T}_{\max } \sim 500^{\circ} \mathrm{F}$ \\
\hline$c$ & sonic velocity, $\mathrm{ft} / \mathrm{sec}$ \\
\hline$C_{p}$ & $\begin{array}{l}\text { specific heat at constant pressure, } \\
\mathrm{ft}-\mathrm{lbf} /\left[\mathrm{lbm}{ }^{\circ} \mathrm{R}\right]\end{array}$ \\
\hline $\mathrm{D}$ & temperature profile label, $\mathrm{T}_{\max } \sim 650^{\circ} \mathrm{F}$ \\
\hline$d$ & tube diameter, in. \\
\hline $\mathrm{D} / \mathrm{A}$ & digital-to-analog conversion \\
\hline $\mathrm{dB}$ & decibel \\
\hline & direct current \\
\hline
\end{tabular}




\begin{tabular}{|c|c|c|c|}
\hline $\mathrm{f}_{0}$ & $\begin{array}{l}\text { minimum frequency in phase-modulated } \\
\text { wave, } \mathrm{Hz}\end{array}$ & $\begin{array}{l}T_{1} \ldots 6 \\
U\end{array}$ & $\begin{array}{l}\text { thermocouple readings along tube, }{ }^{\circ} \mathrm{F} \\
\text { longitudinal velocity, } \mathrm{ft} / \mathrm{sec}\end{array}$ \\
\hline i & general index & $U_{a v g}$ & radial average of longitudinal velocity, $\mathrm{ft} / \mathrm{sec}$ \\
\hline $\mathrm{j}$ & $\sqrt{-1}$ & $U_{\text {creep }}$ & creep flow velocity at tubing wall, $\mathrm{ft} / \mathrm{sec}$ \\
\hline $\mathrm{J}_{0}$ & zeroth order Bessel function & $U_{\text {slip }}$ & slip velocity at the wall \\
\hline$J_{1}$ & first order Bessel function & $U_{s s}$ & steady-state flow velocity, $\mathrm{ft} / \mathrm{sec}$ \\
\hline $\mathrm{J}_{2}$ & second order Bessel function & $V$ & entrapped transducer volume, in $^{3}$ \\
\hline $\mathrm{K}$ & polytropic density proportionality constant & $V_{e}$ & effective volume of a model node \\
\hline $\mathrm{K}^{\prime}$ & $\begin{array}{l}\text { polytropic temperature proportionality } \\
\text { constant }\end{array}$ & $x$ & longitudinal coordinate, in. \\
\hline$L$ & tube length, in. & $z$ & independent variable for Bessel equation \\
\hline lsb & least significant bit & $\alpha$ & shear wave number \\
\hline $\mathrm{M}(x)$ & constant of integration, energy equation & $\gamma$ & ratio of specific heats \\
\hline $\mathbf{n}$ & number of computational nodes & $\Gamma_{\mathrm{p}}$ & propagation factor \\
\hline $\mathrm{N}(x)$ & constant of integration, momentum equation & $\delta f$ & frequency resolution, $\mathrm{Hz}$ \\
\hline $\mathrm{N}_{\mathrm{h}}$ & $\begin{array}{l}\text { number of harmonics in phase-modulated } \\
\text { wave }\end{array}$ & $\begin{array}{l}\varepsilon \\
\eta\end{array}$ & $\begin{array}{l}\text { ratio of slip distance to mean free path } \\
\text { thermal conductivity, } \mathrm{lbf} /\left[\mathrm{sec} /{ }^{\circ} \mathrm{R}\right]\end{array}$ \\
\hline$P$ & pressure, psf & $\vartheta$ & slip distance, in. \\
\hline$P_{\text {cold }}$ & pressure at cold end of tube, psf & $\kappa$ & Knudsen number \\
\hline$P_{h o t}$ & $\begin{array}{l}\text { pressure at hot end of tube, psf } \\
\text { pressure at transducer, psf }\end{array}$ & & $\left(K=1.7725 \sqrt{R_{g}} \frac{\mu}{R} \sqrt{\frac{(T+495.7)}{p}}\right)$ \\
\hline $\begin{array}{l}\mathrm{P}_{\mathrm{r}} \\
P_{0}\end{array}$ & $\begin{array}{l}\text { Prantl number } \\
\text { pressure at surface, psf }\end{array}$ & $\kappa_{p}$ & $\begin{array}{l}\text { rarefied flow correction term in momentum } \\
\text { equation }\end{array}$ \\
\hline $\begin{array}{l}\bar{P}_{0} \\
R\end{array}$ & $\begin{array}{l}\text { mean pressure in tubing, psf } \\
\text { tube radius, in. }\end{array}$ & $\kappa_{0}$ & $\begin{array}{l}\text { Knudsen number based on mean flow } \\
\text { properties }\end{array}$ \\
\hline$r$ & radial coordinate, in. & $\lambda$ & $\begin{array}{l}\text { mean free path of the fluid molecules, } \\
\text { microns }\end{array}$ \\
\hline$R_{g}$ & universal gas constant, $\mathrm{ft}-1 \mathrm{bf} /\left[\mathrm{lbm}{ }^{\circ} \mathrm{R}\right]$ & $\mu$ & dynamic viscosity, $1 \mathrm{bm} /[\mathrm{ft} / \mathrm{sec}]$ \\
\hline $\begin{array}{l}\mathrm{Sig}_{\mathrm{t}} \\
\mathrm{SPL}\end{array}$ & $\begin{array}{l}\text { broadband waveform } \\
\text { sound pressure level, } \mathrm{dB}\end{array}$ & $\mu_{0}$ & $\begin{array}{l}\text { local steady-state bulk viscosity in the } \\
\text { tubing, } 1 \mathrm{bm} /[\mathrm{ft} / \mathrm{sec}]\end{array}$ \\
\hline$T$ & temperature, ${ }^{\circ} \mathrm{R}$ & $\xi$ & polytropic expansion parameter \\
\hline$t$ & time, sec & $\pi$ & the constant $(\pi \approx 3.141726)$ \\
\hline$T_{\text {cold }}$ & temperature at cold end of tube, ${ }^{\circ} R$ & $\rho$ & density, $\mathrm{lbm} / \mathrm{ft}^{3}$ \\
\hline$T_{\text {hot }}$ & temperature at hot end of tube, ${ }^{\circ} \mathrm{R}$ & $\rho_{0}$ & mean density, $1 \mathrm{bm} / \mathrm{ft}^{3}$ \\
\hline$T_{L}$ & temperature at transducer, ${ }^{\circ} \mathrm{R}$ & $\Psi$ & rarefied flow static response parameter \\
\hline$T_{w}$ & wall temperature, ${ }^{\circ} \mathrm{R}$ & $\begin{array}{l}\omega \\
\partial U \\
\end{array}$ & radian frequency, $1 / \mathrm{sec}$ \\
\hline$T_{0}$ & temperature at surface, ${ }^{\circ} \mathrm{R}$ & $\overline{\partial r_{w}}$ & velocity gradic \\
\hline
\end{tabular}




\section{Introduction}

With development of advanced hypersonic vehicle concepts, reliable measurement of onboard trajectory parameters from pneumatic sensors is highly desirable, but measurement of aerodynamic properties on hypersonic vehicles presents formidable challenges. The hostility of the sensing environment precludes intrusion into the flow, and measurements must be obtained via remote sensors. This hostile environment requires using sizable lengths of pneumatic tubing to transmit pressure from the surface to the remotely located transducer. For hypersonic conditions, nominal spectral distortion and acoustical resonance affect the measurements. In addition, large temperature $(T)$ gradients induced by boundary-layer heating induce molecular effects where gas molecules adjacent to the tube wall creep from the cold end of the tube to the hot end. Furthermore, under low pressure conditions, such as those experienced at very high altitudes, the tube flow becomes so rarefied that the fluid slips at the tube wall.

To date, no theoretical model describing influence of these rarefied flow phenomena on pressure measurements is available in the scientific literature. To generally quantify the dynamic behavior of pressure sensors for these hypersonic conditions, NASA Dryden Flight Research Center, Edwards, California, initiated research to develop an accurate frequency response model that is mathematically invertible. That is, given the measured pressure at the pressure sensor, the model could predict the pressure input which occurred at the surface. This invertible model could compensate for pneumatic measurement distortions which cannot be mitigated by the layout of the pneumatic hardware.

This paper describes a general dynamic response model for pressure sensors and applies to continuum and rarefied flow conditions. The model allows for large temperature gradients on the order of $1000^{\circ} \mathrm{R} / \mathrm{ft}$ with a maximum Knudsen number, $\kappa$, of approximately 0.60 . The model was verified using steady-state and dynamic laboratory experiments. Test results and regimes of applicability are also presented. Finite difference methods were not desirable for this application. Instead, the equations of energy and motion are decoupled and reduced to a one-dimensional boundary value problem. The boundary value equations are solved assuming that along a small element gas properties remain constant, and a fundamental solution is developed for this small element. Then, the fundamental solution is used as a building block for a recursive solution method which allows for complex geometries where fluid properties and tubing geometry can vary longitudinally. The problem is solved recursively starting at the transducer end and working toward the surface end of the tube. Using these recursive formulae, solutions for arbitrary geometries and longitudinal temperature profiles can be constructed. The resulting model is fully invertible.

\section{Background}

For full continuum flow which occurs at moderate pressure levels, fluid viscosity causes the gas to stick at the wall, resulting in the classical no-slip boundary condition. On the other hand for rarefied flow conditions, molecular effects become important, and the no-slip boundary condition is no longer valid (ref. 1). For rarefied flow conditions, fluid elements do not stick to the wall as they would in continuum flow. Instead, fluid elements slip along the wall, resulting in a flow regime that is referred to as slip flow. The magnitudes of the molecular effects are proportional to ratio of the molecular mean free path to the characteristic scale length of the flow - Knudsen number. Values of Knudsen numbers less than 0.01 indicate that the flow conditions are continuum and that molecular effects may be ignored. Values between 0.01 and 1.0 indicate the slip flow regime where the flow has elements of continuum and molecular dynamics. Values exceeding 1.0 indicate a free-molecule flow regime, and continuum affects can be ignored.

The mean free path of the fluid molecules, $\lambda$, is the average distance that each fluid particle travels between successive collisions with other fluid particles. If characteristic scale of the system were the tube radius, then the Knudsen number can be approximated by the expression given in equation (1) (ref. 1).

$$
\kappa \approx \sqrt{R_{g} \pi} \frac{\mu}{R} \frac{\sqrt{T}}{P}
$$

where $\pi$ is the constant, $\mu$ is the dynamic viscosity, $R_{g}$ is the universal gas constant, $R$ is the tube radius, $T$ is the temperature, and $P$ is the pressure.

Clearly, Knudsen number is an inverse function of pressure. Thus, rarefied flow phenomena are inherently associated with high Knudsen numbers. For conventional aeronautical applications, the Knudsen 
numbers are always below 0.01 . For hypersonic and orbital applications, Knudsen numbers from 0.05 to 0.20 can be obtained along the flight profile at very high altitudes. Figure 1 illustrates these flow regimes.

The problem of predicting tube flow dynamics has been studied extensively. For nonrarefied, constant temperature conditions, Iberall developed a spectral technique for predicting response lags for sinusoidal inputs and lightly damped configurations (ref. 2). Lamb adapted the work of Iberall to predict step-input rise times for highly damped configurations (ref. 3). Lamb's theory was also applied to predicting steady-state delays for constant ramp inputs. Schuder, et al., (ref. 4) and Hougen, et al., (ref. 5) developed closed form frequency domain solutions for simple tubing geometries and constant wall temperatures. Bergh, et al., (ref. 6) extended the analyses of references 4 and 5 , to develop a recursion formula for complex geometries. The work detailed in reference 6 is the state-of-the-art for predicting tubing responses to constant wall temperatures, $T_{w}$, and continuum flow. Tijdeman extends the model boundary condition to allow for high-speed surface cross flow (ref. 7). He also presents a succinct summary of existing tube response theories (ref. 8).

Parrot, et al., investigated the dynamic transmission of sound in a simple geometry tube which was subjected to very large temperature gradients (ref. 9). These tests were performed for ambient pressure levels, and rarefied flow effects were not considered. Knudsen (ref. 10) and Kennard (ref. 1) investigated tube flow for rarefied conditions with large temperatures. These analyses, however, have been performed only for simple geometries and for steady-flow conditions. A review of the literature did not reveal an unsteady response model. References 1 and 10 present excellent overviews of theoretical and empirical results for steady-state tube flows in rarefied conditions.

Along an unequally heated gas boundary, Maxwell's kinetic theory (ref. 1) predicts that gas molecules originating in the hot region of the tube have higher kinetic energy than molecules originating from the cold region. As a result, such molecules recoil more strongly than molecules from the cold side of the tube. The net result is that the gas acquires a longitudinal momentum in the hotter direction. This net momentum gain causes the gas molecules at the wall to creep from the cold end to the hot end of the tube. To balance this creep, gas molecules in the center of the tube must migrate toward the colder end of the tube. This opposing flow equilibrium results in establishment of a steady-state pressure gradient. The cold region of the tube has a lower pressure than the hot region, and no net crosssectional flow exists in the tube.

In his analyses, Maxwell determined that in the free molecular limit the normalized ratio of the creepinduced pressure gradient was one-half of the normalized temperature gradient. For example,

$$
\frac{\partial P}{P}=\frac{1}{2} \frac{\partial T}{T}
$$

where $\partial P / \partial x$ is the induced longitudinal pressure gradient, $P$, is the nominal pressure in the tube, $\partial T / \partial x$ is the longitudinal temperature gradient, and $T$ is the nominal temperature in the tube. For conditions which lie somewhere between the free molecular regime and continuum flow, the pressure gradient induced by longitudinal temperature gradients is less than one-half and is a strong function of Knudsen number (refs. 1 and 10).

For slip flow conditions, the primary molecular effect is the fluid movement at the wall boundary. The fluid velocity at the wall boundary can be decomposed into two parts: slip velocity and thermomolecular creep velocity. The slip velocity, $U_{\text {slip }}$, (ref. 1) is proportional to the shear stress at the wall, and for laminar flow may be written in terms of the velocity gradient as

$$
U_{s l i p}=-\vartheta \frac{\partial U}{\partial r_{w}}
$$

where $U$ is longitudinal velocity, and $r$ is the radial coordinate.

The parameter, $\vartheta$, is referred to as the slip distance and is dimensioned in units of length. The slip distance is on the order of the mean free path of the flow (ref. 1). The ratios of slip distance to mean free path for various channel materials and gases are tabulated in references 1 and 9. For the flow of air over machined brass or steel, the ratio is 0.995 ; for air flowing over glass, the ratio is 1.24 .

The molecular creep velocity is one of the more peculiar phenomenon which occurs at low pressure. 
Kennard shows that the creep velocity is directly proportional to the longitudinal temperature gradient and inversely proportional to the local pressure (ref. 1). Its magnitude can be approximated by the expression

$$
U_{\text {creep }}=\frac{3}{4} \frac{\mu \partial T / \partial x}{\rho_{0} T(x)} \approx \frac{3}{4} \frac{\mu_{0} R_{g}}{\bar{P}_{0}} \frac{\partial T}{\partial x_{w}}
$$

where $\mu_{0}$ and $\bar{P}_{0}$ are longitudinal average viscosity and pressure in the tubing. At the wall boundary, the velocity is the sum of the two terms. For example,

$$
\begin{aligned}
U(x, R, t) & =U_{\text {slip }}+U_{\text {creep }} \\
& \approx-\vartheta \frac{\partial U}{\partial r}+\frac{3}{4} \frac{\mu_{0} R_{g}}{\widetilde{P}_{0}} \partial T / \partial x
\end{aligned}
$$

For slip flow conditions, the condition given by equation (5) replaces the traditional no-slip, $U(x, R, t)=0$, boundary condition used in continuum fluid mechanics. Other than this modification, the classical equations of fluid motion apply in this flow regime (refs. 1 and 11).

\section{Mathematical Analysis}

This section presents the mathematical analyses used to develop the rarefied flow dynamic model. The boundary value equations describing the pressure wave propagation in the tube are presented first. Next, a recursive solution method for these boundary value equations is stated. Finally, the steady-state behavior of the frequency response model is aralyzed. Appendixes $\mathrm{A}, \mathrm{B}$, and $\mathrm{C}$ present detailed development of all mathematical analyses.

\section{Derivation of the Boundary Value Equations}

The model is derived from the unsteady, threedimensional Navier-Stokes equations which are linearized using a small perturbation assumption. The energy equation is decoupled from the equations of momentum and continuity, assuming the longitudinal wave expansion process within the tube is polytropic (refs. 1-7, 11, and 12). For a polytropic process, the relationship between pressure, temperature, and density is described by the simple model

$$
P=\mathrm{K} \rho^{\xi}=\mathrm{K}^{\prime} T^{\xi /(\xi-1)}
$$

where

$$
\begin{aligned}
& P=\text { pressure } \\
& \mathrm{K}=\text { proportionality constant for density } \\
& \mathrm{K}^{\prime}=\text { proportionality constant for temperature } \\
& \xi=\text { polytropic expansion parameter } \\
& \rho=\text { density } \\
& T=\text { temperature }
\end{aligned}
$$

Limiting values for $\xi$ are given by $1<\xi<\gamma$ where $\xi=1$ corresponds to an irreversible isothermal expansion process, and $\xi=\gamma$ corresponds to a completely reversible isentropic expansion process. Using the polytropic flow assumption allows decoupling the energy equation from the equations of momentum and continuity without loss of generality. Appendix A presents the variation of $\xi$ as a function of input frequency and the fundamental flow parameters. The momentum equation is integrated to give the local flow velocity in terms of the longitudinal pressure gradient. The slip flow boundary condition (eq. (5)) is used to solve for the constant of integration.

The result is averaged over the cross-section of the tube to give a radially averaged flow equation. The resulting equation is coupled with the radially averaged continuity equation to develop a wave equation which describes the pressure propagation in the tube for rarefied flow conditions. Similar arguments are used to develop a downstream longitudinal boundary condition. The upstream pressure is assumed to be a prescribed input. The resulting boundary value equations,

wave equation

$$
\frac{\partial^{2} P(x)}{\partial x^{2}}=\left[\omega \frac{\Gamma_{p}}{c}\right]^{2} P(x)
$$

downstream boundary condition

$$
\begin{aligned}
\frac{\partial P}{\left.\partial x\right|_{x=L}} & =-\frac{\omega^{2} \Gamma_{p}^{2}}{c^{2}} \frac{V}{A_{c}} P_{L} \\
P(0, \omega) & \equiv P_{0}(\omega)
\end{aligned}
$$


propagation velocity

$$
U_{a v g}(x)=\frac{\frac{\partial P}{\partial x}}{\left[j \omega \frac{\xi}{\gamma} \Gamma_{p}^{2} \rho_{0}\right]}
$$

and upstream pressure input, $P_{0}(\omega)$, are prescribed. (See appendix A.)

$$
\Gamma_{p}
$$

$$
\equiv \sqrt{\frac{\gamma}{\left\{\kappa_{p}\left(\mathrm{~J}_{2}[\alpha]+\vartheta \frac{\alpha}{R} \mathrm{~J}_{1}[\alpha]\right)+\mathrm{j} \omega \frac{3}{4} \frac{\mu_{0}}{\bar{P}_{0}} \frac{\xi-1}{\xi} \frac{2}{\alpha} \mathrm{J}_{1}[\alpha]\right\}}}
$$

where

$$
\begin{array}{ll}
V & =\text { transducer volume } \\
c & =\text { sonic velocity } \\
A_{c} & =\text { tube cross-sectional area } \\
L & =\text { tube length } \\
P_{L} & =\text { pressure at the transducer } \\
\rho_{0} & =\text { surface pressure } \\
\kappa_{p} & =\text { rarefied flow correction factor for the bulk } \\
U_{a v g} & =\text { longitudinally averaged velocity } \\
\omega & =\text { radian frequency } \\
\alpha & =\text { shear wave number } \\
\frac{\partial P}{\partial x} & =\text { longitudinal pressure gradient } \\
\bar{P}_{0} \quad=\text { mean pressure in the tubing }
\end{array}
$$

The propagation factor, $\Gamma_{p}$, given by equation (10) is a new result not presently available in the scientific literature. This factor accounts for molecular effects and frictional damping in the tube and is a generalization of the work presented in references 6 and 7. Parameters $\mathrm{J}_{0}$ and $J_{1}$ are the Bessel functions (ref. 13) of the zeroth and first orders.

\section{Boundary Value Equations Solution}

The boundary value equations are solved in the frequency domain. Temperature and gas properties are assumed to remain constant along the length of the tube to give a fundamental solution where the complex spectra are given as a function of the sensor geometry, the frequency of the input sinusoid, and the propagation factor. This solution is detailed in appendix B.

$$
P_{L}(\omega)
$$

$$
\left.=P_{0}(\omega)\left[\frac{1}{\cosh \left[\omega \Gamma_{p}\right.} \frac{\left.\frac{L}{c}\right]+\frac{V \omega \Gamma_{p}}{A_{c} c} \sinh \left[\omega \Gamma_{p}\right.}{\frac{L}{c}}\right]\right]
$$

This fundamental solution for constant temperature and tube radius is used as a building block for complex geometries in which the wall temperature, fluid properties, and tube geometry vary longitudinally. For longitudinal variations within the tube, the problem is solved recursively starting at the transducer end and working toward the surface (external) end of the tube. As developed in appendix $\mathrm{B}$, the solution at the ith node is

$$
\begin{aligned}
& P_{\mathrm{i}}(\omega) \\
& =\frac{P_{\mathrm{i}-1}(\omega)}{\cosh \left[\omega \Gamma_{p_{\mathrm{i}}} \frac{L_{\mathrm{i}}}{c_{\mathrm{i}}}\right]+\omega \frac{V_{e_{\mathrm{i}}}}{A_{c_{\mathrm{i}}}} \frac{\Gamma_{p_{\mathrm{i}}}}{c_{\mathrm{i}}} \sinh \left[\omega \Gamma_{p_{\mathrm{i}}} \frac{L_{\mathrm{i}}}{c_{\mathrm{i}}}\right]}
\end{aligned}
$$

where the effective volume, $V_{e}$, accounts for the entrapped volume at the $i$ th node plus the impedance of the downstream tubes and volumes. The general end-toend frequency response is given as the complex product of the frequency responses at the individual nodes.

$$
\begin{aligned}
& \frac{P_{L}(\omega)}{P_{0}(\omega)}=\frac{P_{1}(\omega)}{P_{0}(\omega)} \frac{P_{2}(\omega)}{P_{1}(\omega)} \cdots \frac{P_{\mathrm{n}-1}(\omega)}{P_{\mathrm{n}-2}(\omega)} \frac{P_{\mathrm{n}}(\omega)}{P_{\mathrm{n}-1}(\omega)} \\
& =\prod_{\mathrm{i}=1}^{\mathrm{n}} \frac{1}{\cosh \left[\omega \Gamma_{p_{\mathrm{i}}} \frac{L_{\mathrm{i}}}{c_{\mathrm{i}}}\right]+\omega \frac{V_{e_{\mathrm{i}}}}{A_{c_{\mathrm{i}}}} \frac{\Gamma_{p_{\mathrm{i}}}}{c_{\mathrm{i}}} \sinh \left[\omega \Gamma_{p_{\mathrm{i}}} \frac{L_{\mathrm{i}}}{c_{\mathrm{i}}}\right]}
\end{aligned}
$$


Equations (11) through (13) allow for a finite-element solution of the boundary value problem. These equations are used to generate the frequency response solutions for complex geometries or for longitudinal temperature distributions. If molecular effects are ignored, and a constant temperature profile is assumed, these equations are mathematically identical to the recursion formulae developed in references 6 and 7 .

\section{Steady-State Response of the Dynamic Model}

Maxwell's analysis predicts that in the presence of large temperature gradients and rarefied flow, the equilibrium pressure gradient in the tube is nonzero. The model (eqs. (10) - (13)) exhibits a similar steadystate behavior. Equilibrium behavior of the model for rarefied flow conditions is best understood by looking at the momentum equation at a given longitudinal crosssection. The normalized steady pressure gradient can be written as a function of Knudsen number and normalized temperature gradient.

$$
\frac{\frac{\partial P}{\partial x}}{P} \approx \frac{6 \kappa_{0}^{2}}{\pi\left(1+4 \varepsilon \kappa_{0}\right)} \frac{\frac{\partial T}{\partial x}}{T}
$$

(See appendix C.) In equation (14), $\varepsilon$ is the ratio of the slip distance to the mean free path and, for this analysis, can be assumed to be unity. Equation (14) is extremely important because Maxwell predicts that in the freemolecule limit (Knudsen numbers approaching infinity)

$$
\left[\frac{\frac{\partial P}{\partial x}}{P}\right]+\left[\frac{\frac{\partial T}{\partial x}}{T}\right]=\frac{1}{2}
$$

Clearly, equation (6) does not approach a limit. Therefore, the model has an upper boundary for which the slip flow assumptions are valid. Because it is extremely difficult to conduct controlled dynamic experiments under rarefied flow conditions, steady-state behavior of the model is the only feasible means of evaluating the validity and range of applicability for the slip flow assumptions used in deriving the dynamic model. Empirical validation of the model is described next.

\section{Experimental Apparatus and Procedures}

The assumptions used in deriving the model and the flow regimes to which the model applies were evaluated using a series of laboratory tests. First, dynamic frequency response tests were performed for continuum flow conditions at room temperature and in the presence of large temperature gradients. Results of these tests demonstrated the validity of the polytropic energy analysis and the recursive formulation for temperature gradients in the tubing. Next, steady-state response tests were performed for rarefied flow conditions. These tests were used to verify the slip flow assumptions used in deriving the boundary value equations and to establish a regime of validity for the model. Results from these experimental tests are compared to analytical predictions in the Results and Discussion section.

\section{Dynamic Frequency Response Tests}

Frequency response measurements were gathered using a test plate mounted at the end of the sound chamber. Figure 2(a) presents a schematic of the test configuration, and figure 2(b) shows an overview of the test equipment layout. Reference sound pressure levels impinging on the plate were measured by a constant current piezoelectric microphone mounted flush to the plate. The response of a test configuration, which consisted of a flush surface port and a section of brass tubing, was measured by an identical microphone mounted in a housing at the end of the tubing.

Frequency response was evaluated by comparing the output of the test microphone to the output of the reference microphone. For these tests, a broad-band wave form was generated by a microcomputer outfitted with a 12-bit digital-to-analog (D/A) conversion board, amplified with commercial stereo equipment, and used to excite a large speaker inserted in an anechoic chamber. The speaker is shown in the sound chamber in figure 2(c). Speaker volume was controlled using a voltage attenuator on the output voltage from the microcomputer. By changing the speaker sizes and output roll off, frequency ranges from approximately 0.50 to $2000 \mathrm{~Hz}$ could be accurately evaluated.

High-temperature gradients were induced by a heater made from a 3/4-in. diameter aluminum rod heated with electrical resistance heating tape. The rod was bored with a hole its entire length, and the section of brass tubing to be evaluated was press-fit into the hole. The temperature of the heating rod was regulated using a temperature controller and a feedback thermocouple. One end of the brass tube was soldered flush to the surface port in the test plate, and the other end was fitted to the microphone housing. Copper-constant (Type T) 
thermocouples (TC) were used to sense the temperature at the surface port, the microphone housing, and four points along the length of the tube. Thermocouples were joined to a single electronic reference junction, and their readings were selectable using a rotary switch. In the ranges tested, the estimated accuracy of the thermocouple measurements was approximately $\pm 2{ }^{\circ} \mathrm{F}$. These ranges were based on the manufacturer's specifications. Figure 2(c) also shows the heater configuration and the test plate arrangement.

Output signals from the reference and test microphones were amplified and sampled at $24 \mathrm{kHz}$ by a 16-bit analog-to-digital (A/D) conversion board in the microcomputer. Direct current $(\mathrm{dc})$ offsets in the microphone outputs were removed by alternating current (ac) coupling the microphone outputs to give a minimum response frequency of approximately $0.05 \mathrm{~Hz}$. The flat frequency responses of the microphones and signal conditioning extended to well beyond $10 \mathrm{kHz}$. At nominal sound volume levels, measurements showed that the sound pressure level (SPL) in the chamber was approximately $145 \mathrm{~dB}$ (7.5 psf). This SPL is well within the linear response regions of the reference and test microphones. In the linear range these microphones have an unamplified response sensitivity of $10.4 \mu$ volt/psf. Using amplifier gains settings of 10 and a full-scale $A / D$ range of \pm 1 volt, the nominal resolution of the microphone least significant bit (lsb) was approximately $0.0029 \mathrm{psf} / \mathrm{sb}$.

The broad-band wave form used to excite the speaker was generated by a nonlinear phase-modulated cosine series of the form

$$
\operatorname{Sig}_{t}=\sum_{i=0}^{N_{h}} \cos \left[2 \pi\left(f_{0}+i \delta f\right) t+\frac{i^{3} \pi}{N_{h}}\right]
$$

where $f_{0}$ is the minimum frequency in the wave form, $\delta \mathrm{f}$ is the spacing between harmonics, $N_{h}$ is the number of harmonics in the wave form, $t$ is time, and $N_{h} \delta f$ is the maximum excited frequency. The nonlinear phase modulation ensures that energy is distributed uniformly in the time and frequency domains (ref. 14) and that the wave form will be physically realizable.

Figures 3(a) and 3(b) show the time history and spectra of a sample wave form. Large sample runs (typically 100,000 data points) were taken for each test, and an ensemble of coarse transfer functions was evaluated using a fast Fourier algorithm with a 4096-point data window. The coarse transfer functions resulting from each data window along the time history were ensemble averaged for the entire data record. Ensemble averaging helps to mitigate the affects of resolution and random measurement errors and produces a clean transfer function output.

For each data run, ambient pressure levels were recorded with a hand-held manometer, and a baseline data set at ambient temperature levels was taken. The heater was turned on with the required setting selected on the temperature controller, and the system was allowed to stabilize. The frequency response data were obtained, and the temperature readings at each of the six thermocouples was recorded. The temperature was then raised to the next condition and allowed to stabilize. After data at the maximum temperature which could be obtained by the system, approximately $650^{\circ} \mathrm{F}$, was recorded, the system was allowed to cool. Next, the tests at lower temperature settings were repeated.

\section{Steady-State Response Tests for Rarefied Flow Conditions}

Figure 4(a) shows the apparatus layout for the steadystate response tests. These tests were used to verify the steady-state response of the analytical model for rarefied conditions and to evaluate the upper limit of Knudsen numbers for which the model is valid. As mentioned in the Background section and appendix C, the model theory predicts that in the presence of large temperature gradients and rarefied flow conditions, gas adjacent to the tube wall creeps from the colder region to the hotter region. The result is an opposing flow which establishes a steady-state pressure gradient within the tube, with the cold region of the tube reading lower temperatures than the hot region. These tests reproduced those conditions.

The steady-state response tests were performed in an evacuated vacuum oven. Here, 3/4-in. diameter aluminum rods were bored with holes, and an assortment of brass tubes of varying diameters and lengths was press-fit into the holes. As the oven was heated, the aluminum rods provided a thermal mass to distribute the heat evenly along one end of the tubing. Type $\mathrm{T}$ thermocouples bonded to each end of the tube were used to sense the absolute temperatures and the temperature gradient along the tube. As before, the thermocouples were joined to a single electronic 
reference junction, and their readings were selectable using a rotary switch. Accuracies were similar to those values obtained in the frequency response tests. Thermocouple wire was passed from the vacuum chamber to the thermocouple reference panel using a hermetically sealed thermocouple fitting on the back of the oven.

The heated end of the tube was open to the oven chamber, and the cold end was hermetically bonded to a compression fitting that allowed access to the tube from outside of the evacuated vacuum chamber. The chamber pressure was measured using a highly accurate Vernier manometer, and the pressure differential in the tubing was measured using a differential McLeod gauge (ref. 15). The oven vent was branched off to the manometer and to the reference side of the McLeod gauge and pressure valve was used to isolate the two instruments when readings were being taken. A close up of the test configuration showing the attached thermocouples and the pressure fittings is shown from the front view in figure 4(b) and from the rear view in figure 4(c).

Tests were conducted by first recording the zero differential pressure in the tube at ambient temperature and pressure. The heater was turned on with the required setting selected on the temperature controller. Next, the system temperature was allowed to stabilize. Then, the chamber was evacuated to the approximate desired pressure level, and the system was sealed. The hot and cold end temperatures were recorded using the thermocouples. At this point, the chamber pressure was recorded using the Vernier manometer, and the differential pressure in the tubing was recorded using the McLeod gauge. At the end of each data point, the temperature setting was maintained constant. In addition, chamber pressure was adjusted to the new desired value. The system was allowed to stabilize, and a new set of readings was taken.

For each temperature setting, approximately 30 pressure test points were recorded, starting at the lowest pressure and working toward higher pressures. At the end of each set of runs, the system was vented and allowed to cool to ambient temperatures. Then, a new zero differential pressure reading was taken. The pre- and post-test zero readings were used to correct the differential pressure measurement for bias offsets in the McLeod gauge. Standard accuracy for a Vernier manometer is on the order of $10-25$ microns of mercury (0.030-0.080 psf). The accuracy of the differential McLeod gauge is on the order of 5-10 microns of mercury (0.015-0.030 psf) (ref. 15).

\section{Results and Discussion}

Results of the frequency response tests are described first. Comparisons to the analytical model for selected geometries and temperature profiles are presented. Next, the results of the steady-state response tests are presented. These data were parameterized as a function of Knudsen number and compared to the predictions of the analytical model. From the comparisons, a range of valid Knudsen numbers for the model has been established.

\section{Frequency Response Tests}

Sixteen data runs were performed. There were four temperature profiles for each of the two tube geometries. Each test was repeated twice. All of the tests were performed using a broad-band wave form with spectral energy from 10 to $4000 \mathrm{~Hz}$. Table 1 presents the frequency response test matrix, including the tube geometry and the temperature readings for each of the six thermocouples bonded to the tube. For these tests, the maximum attainable heater temperature was approximately $650^{\circ} \mathrm{F}$. This configuration resulted in a maximum temperature gradient of approximately $1300^{\circ} \mathrm{F} / \mathrm{ft}$.

Temperature readings from the repeated data runs were averaged and interpolated to give idealized temperature profiles along the length of the tube. These profiles, labeled A, B, C, and D, are presented in figures 5(a) and 5(b) for the 0.066- and 0.033-in. diameter tubes. Using these idealized temperature profiles, the theoretical frequency responses of the test geometries were evaluated using the recursive formula of equation (13).

The computations were basically insensitive to the number of grid points. This insensitivity is illustrated in figure 6 where the frequency response of the 0.066-in. diameter tube was evaluated, assuming temperature profile $\mathrm{D}$. These computations were performed with 5 , $10,20,50$, and 100 equally spaced nodes. Beyond 10 elements, little difference exists in the computations. Beyond 20 elements, the solutions are virtually identical. Thus for this analysis, 20 solution elements 
were used. The resulting calculations are shown in figure 7 (a) for the 0.066 -in. diameter tube and figure 7(b) for the 0.033-in. diameter tube for temperature profiles $\mathrm{A}, \mathrm{B}$, and $\mathrm{C}$.

The overall effect of increasing temperature gradients is an increase of the phase delay of the response and a shift of the spectral harmonics to higher frequency and lower magnitude. This effect is verified extremely well by the data. The results are presented in figures 8 and 9 for the 0.066- and 0.033-in. diameter geometries. Here, the model calculations are overplotted against the transfer function data averaged from the repeated runs for the various temperature profiles of figure 5 . The agreement is excellent for all of the cases. Up to the approximately $2000-\mathrm{Hz}$ limits of the data, the frequency response is predicted to within the noise limits of the basic measurement. That is, the locations of the harmonics are predicted to within $1-2 \mathrm{~Hz}$, and the spectral magnitudes are predicted to within $2 \mathrm{~dB}$ along the entire frequency band. Clearly, the energy analysis and recursive formulation are entirely valid for the temperature ranges presented.

\section{Steady-State Response Tests for Rarefied Flow} Conditions

These steady-state response tests were performed to assess the upper limit of Knudsen number for which the rarefied flow terms in the model are valid. For the steady-state tests, tube diameters from 0.092- to 0.014-in., temperature gradients as high as $950^{\circ} \mathrm{F} / \mathrm{ft}$, and chamber pressures as low as 100 microns of mercury $\left(0.28 \mathrm{lbf} / \mathrm{ft}^{2}\right)$ were tested. The resulting Knudsen numbers varied from zero to approximately 10. Table 2 presents the test matrix which was investigated.

Rearranging equation (14) to collect Knudsen number gives

$$
\frac{T}{P} \frac{\partial P / \partial x}{\partial T / \partial x} \approx \frac{6 \kappa_{0}^{2}}{\pi\left(1+4 \varepsilon \kappa_{0}\right)}
$$

Equation (17) suggests a manner to display the results of the steady-state response tests. Approximating the derivatives in equation (17) by differences yields

$$
\frac{\frac{\partial P / \partial x}{P}}{\frac{\partial T / \partial x}{T}} \approx \frac{\frac{P_{h o t}-P_{\text {cold }}}{\frac{1}{2}\left(P_{\text {hot }}+P_{\text {cold }}\right)}}{\frac{T_{\text {hot }}-T_{\text {cold }}}{\frac{1}{2}\left(T_{\text {hot }}+T_{\text {cold }}\right)}} \equiv \Psi
$$

These data can be collapsed to a single curve by plotting the rarefied flow static pressure parameter, $\Psi$, against Knudsen number averaged over the hot and cold ends of the tube. These results are plotted in figure 10 along with equation (17) evaluated using 1.0 for the proportionality constant, $\varepsilon$.

For pressures below 350 microns $\left(0.28 \mathrm{lbf} / \mathrm{ft}^{2}\right)$, the vacuum oven chamber pressure was difficult to maintain, and the data are somewhat suspect. However, these data appear to approach the free-molecule limit of 0.5 (eq. (2)). The comparison to the model is excellent for Knudsen numbers up to approximately 0.6 . Because equation (17) was derived directly from the fundamental solution of the dynamic model (eq. (11)), the model appears valid for most of the slip flow regime. For $\kappa>0.6$, free-molecule affects dominate, and the model rapidly diverges from the data. This Knudsen number is the upper boundary on the model's usefulness. For aeronautical applications, this Knudsen number occurs only under near-orbital conditions (fig. 1).

\section{Concluding Remarks}

Measurement of aerodynamic properties on hypersonic vehicles presents formidable challenges. The hostility of the sensing environment disallows intrusion into the flow. For this reason, measurements must be obtained through remote sensors. In addition, sizable lengths of pneumatic tubing must be used to transmit pressure from the surface to the remotely located transducer. Because pneumatic measurements are necessary to compute vital flight mechanics parameters, such as angle of attack, dynamic pressure, and Mach number, or to evaluate surface pressure distributions, it is essential that the dynamic behavior of tubing transducer measurement configurations be well understood for hypersonic flight conditions. These conditions include high surface temperature gradients and rarefied flow.

This paper develops a general dynamic response model for pressure sensors in high Knudsen number flow with large temperature gradients. The model applies to continuum and rarefied flow conditions and allows large temperature gradients within the pneumatic tubing. The sensor response model is developed from the Navier-Stokes equations and linearized by small perturbations. It decouples the energy equation by 
assuming that the wave expansion in the tube is polytropic.

The model is converted to a one-dimensional boundary value problem by radially averaging flow properties. The boundary value equations are solved in the frequency domain, assuming that the gas properties remain constant along the length of the tube. This fundamental solution is used as a building block for complex geometries in which the fluid properties in the tube vary longitudinally. The problem is solved recursively starting at the transducer end and working toward the surface end of the tube. Using the recursive formula, solutions for arbitrary geometries and longitudinal temperature profiles can be constructed.

The steady-state behavior of the model is analyzed by applying the final value theorem to the recursive equation. The resulting expression is nondimensionalized and written as a function of Knudsen number. The steady-state response function is used to evaluate the regime of applicability of the dynamic model.

The assumptions used in deriving the model and the flow regimes to which the model applies were evaluated using a series of dynamic and steady-state laboratory tests. Dynamic frequency response tests were performed for continuum flow conditions and temperature gradients as large as $1300^{\circ} \mathrm{F} / \mathrm{ft}$. Steady-state response tests were performed for rarefied flow conditions with chamber pressures as low as 100 microns of mercury $\left(0.28 \mathrm{lbf} / \mathrm{ft}^{2}\right)$ and temperature gradients as high as $950^{\circ} \mathrm{F} / \mathrm{ft}$. The resulting Knudsen numbers varied from zero to approximately 10 .

The dynamic frequency response tests demonstrated the accuracy of the polytropic energy analysis, fundamental solution, and recursive formulation for temperature gradients. Increasing temperature gradients resulted in an increase in the phase delay of the response and a shift of the spectral harmonics to higher frequency and lower magnitude. This effect is verified extremely well by the data. Up to the approximately $2000-\mathrm{Hz}$ limits of the data, the frequency response is predicted to within the noise limits of the basic measurement.

The steady-state response tests verified the slip flow assumptions used in deriving the boundary value equations and established an upper boundary on the applicability of the model. Model comparisons are excellent for Knudsen numbers up to around 0.6. For values of $\kappa>0.6$, free-molecule effects begin to dominate the flow, and the model analyses are no longer valid.

The model represents a fundamental contribution to the understanding of flow behavior at the limits of the continuum flow regime. The model allows instrumentation designers to evaluate the responses of pneumatic systems over a wide range of flow conditions in a general and unified way without having to resort to ad hoc or special case models.

\section{APPENDIX A DEVELOPMENT OF BOUNDARY VALUE EQUATIONS}

This appendix develops the boundary value equations for the mathematical model. The basic strategy is to linearize the fundamental equations of energy, continuity, and momentum by assuming small input perturbations. The partial differential equations are reduced to ordinary differential equations using the Fourier transform, and the boundary value equations are developed in the frequency domain. To account for slip and rarefied flow effects, a slip flow boundary condition is allowed at the tubing walls. The resulting equations are averaged across the cross-section of the tube to give a one-dimensional model. For small tube diameters, no radial pressure gradients exist; therefore, little loss in generality occurs. The energy equation is decoupled from the equations of momentum and continuity by assuming the wave expansion in the tube to be polytropic (ref. 12).

\section{Coordinate Definitions and Basic Assumptions}

The sensor configuration is modeled as a straight cylindrical tube with the internal volume of the pressure transducer attached to its downstream end (fig. A-1). The total tube length is $L$. A longitudinal coordinate, $x$, is defined from the upstream (port) end of the tube, and a radial coordinate, $r$, is defined starting at the center of the tube. At each longitudinal station, the tube has radius, $R$, not necessarily a constant for each longitudinal station. The density and velocity distributions, $\rho(x, r, t), u(x, r, t)$, vary as a function of longitudinal distance down the tube, radial distance 
from the center of the tube, and function of time. The parameter $U$ is the longitudinal velocity. Pressure variations at the surface propagate as longitudinal waves through the connective tubing to the transducer. The wave propagation is damped by frictional aitenuation along the walls of the tubing. When the wave reaches the downstream end of the tubing, it reflects back up the tube and may either damp or amplify incoming pressure waves.

For small tubes, flow occurs only in the longitudinal direction. Assuming that the system is initially at rest and input disturbances are small, the second-order terms are neglected. In addition,

$$
\frac{\partial P}{\partial r}, U \frac{\partial T}{\partial x}, U \frac{\partial P}{\partial x},\left\{\frac{\partial U}{\partial x}\right\}^{2} \ldots \approx 0
$$

The temperature distribution in the tube is assumed to be forced by heat transfer from or to outside sources and sinks. The wall temperature profile, $T_{w}(x)$, is assumed to be prescribed and known a priori. To simplify the analysis at any given longitudinal station, the temperature gradient is assumed to be constant. Thus,

$$
\frac{\partial^{2} T}{\partial x^{2}} \approx 0
$$

Later, because the problem will be cast as a finiteelement solution with a series of piecewise longitudinal temperature variations, this assumption is not too restrictive.

Unlike continuum flow conditions where fluid elements stick to the tubing wall and the classical noslip boundary condition holds, for rarefied flow conditions, the fluid velocity at the wall is not zero. Large temperature gradients can result in the so-called molecular creep effect. This effect is primarily a molecular phenomenon where gas molecules adjacent to the tube wall creep from the cold end of the tube to the hot end. Furthermore, under low-pressure conditions, such as those experienced at very high altitudes, tube flow can become so rarefied that the fluid slips at the tube wall. Modification of this boundary condition makes the rarefied flow problem unique.

\section{Energy Analysis}

Based on the assumptions stated in the previous section, the energy balance is (ref. 6)

$$
\rho_{0} C_{p} \frac{\partial T}{\partial t}=\eta\left[\frac{\partial^{2} T}{\partial r^{2}}+\frac{1}{r} \frac{\partial T}{\partial r}\right]+\frac{\partial P}{\partial t}
$$

where $\eta$ is thermal conductivity of the fluid, $C_{p}$ is the specific heat at constant pressure, $\rho_{0}$ is the nominal density, $T$ is the local temperature, and $P$ is the local pressure. Solving for the time derivative of temperature and taking the Fourier transform of equation (A-3),

$$
T-\frac{\mu}{j \omega \rho_{0} P_{r}}\left[\frac{\partial^{2} T}{\partial r^{2}}+\frac{1}{r} \frac{\partial T}{\partial r}\right]=\frac{P}{\rho_{0} C_{p}}
$$

where the $\mu$ is the dynamic viscosity, and $P_{r}=\frac{\mu C_{p}}{\eta}$ is the Prantl number.

Defining the shear wave number, $\alpha \equiv j \sqrt[3 / 2]{\left(\omega \rho_{0} R^{2}\right) / \mu_{0}}$ and a nondimensional longitudinal coordinate $z=\alpha \frac{r}{R} \sqrt{P_{r}}$ where $\mu_{0}$ is the bulk (radially averaged) viscosity of the fluid. Equation (A-4) becomes

$$
\left[\frac{\partial^{2} T}{\partial z^{2}}+\frac{1}{z} \frac{\partial T}{\partial z}+T\right]=\frac{P}{\rho_{0} C_{p}}
$$

Equation (A-5) is a form of Bessel equation of order zero (ref. 13) and has a general solution of the form

$$
T(x, r)=\mathrm{M}(x) \mathrm{J}_{0}\left[\sqrt{P_{r}} \alpha \frac{r}{R}\right]+\frac{P}{\rho_{0} C_{p}}
$$

where $\mathrm{J}_{0}$ is Bessel function of the zeroth order. The parameter $\mathrm{M}(x)$ is evaluated using the boundary condition at the wall, $T(x, R)=T_{w}(x)$. Thus,

$$
\begin{aligned}
T(x, r)= & {\left[T_{w}(x) \frac{\mathrm{J}_{0}\left[\sqrt{P_{r}} \alpha \frac{r}{R}\right]}{\mathrm{J}_{0}\left[\sqrt{P}_{r} \alpha\right]}\right] } \\
& +\left\{1-\frac{\mathrm{J}_{0}\left[\sqrt{P_{r}} \alpha \frac{r}{R}\right]}{\mathrm{J}_{0}\left[\sqrt{P_{r}} \alpha\right]}\right\} \frac{P(x)}{\rho_{0} C_{p}}
\end{aligned}
$$

Equation (A-7) is now written as a one-dimensional radially averaged 


$$
\begin{aligned}
T_{a v g}(x)= & \frac{\int_{0}^{2 \pi} \int_{0}^{R} T(x, r) r d r d \theta}{\pi R^{2}} \\
= & T_{w}(x)\left\{1+\frac{\mathrm{J}_{2}\left[\sqrt{P_{r}} \alpha\right]}{\mathrm{J}_{0}\left[\sqrt{P_{r}} \alpha\right]}\right\} \\
& -\frac{P(x)}{\rho_{0} C_{p}} \frac{\mathrm{J}_{2}\left[\sqrt{P_{r}} \alpha\right]}{\mathrm{J}_{0}\left[\sqrt{P_{r}} \alpha\right]}
\end{aligned}
$$

where $\mathrm{J}_{2}$ is the second-order Bessel function. Because no radial pressure gradients exist,

$$
\frac{P(x)}{\rho_{0} C_{p}} \approx \frac{R_{g} T_{w}}{C_{p}}=\frac{\gamma-1}{\gamma} T_{w}
$$

where $R_{g}$ is the universal gas constant, and $\gamma$ is the ratio of specific heats. Equation (A-8) is approximated as

$$
T_{a v g}(x)=T_{w}(x)\left\{1+\frac{1}{\gamma} \frac{\mathrm{J}_{2}\left[\sqrt{P_{r}} \alpha\right]}{\mathrm{J}_{0}\left[\sqrt{P_{r}} \cdot \alpha\right]}\right\}
$$

\section{Polytropic Analysis}

To decouple the energy equation from the equations of momentum and continuity, density and temperature are written in terms of pressure by assuming that the wave expansion process in the tube is polytropic (ref. 12).

$$
P=\mathrm{K} \rho^{\xi}=\mathrm{K}^{\prime} T^{\xi /(\xi-1)}
$$

Differentiating equation (A-11) with respect to density

$$
\frac{\partial P}{\partial \rho}=\xi \frac{P}{\rho}=R_{g} T=\frac{\xi}{\gamma} c^{2}
$$

and temperature

$$
\frac{\partial P}{\partial T}=\frac{\xi}{\xi-1} \frac{P}{T}
$$

where $c$ is the local sonic velocity. Differentiating equation (A-10) with respect to temperature gives

$$
\begin{aligned}
\frac{\partial P}{\partial T} & =-\frac{P(x)}{T(x)}\left[\frac{\rho_{0} C_{p} T(x)}{P(x)}\right]\left\{\frac{\mathrm{J}_{0}\left[\sqrt{P_{r}} \alpha\right]}{\mathrm{J}_{2}\left[\sqrt{P_{r}} \alpha\right]}\right\} \\
& \approx-\frac{\gamma}{\gamma-1}\left\{\frac{\mathrm{J}_{0}\left[\sqrt{P_{r}} \alpha\right]}{\mathrm{J}_{2}\left[\sqrt{P_{r}} \alpha\right]}\right\} \frac{P(x)}{T(x)}
\end{aligned}
$$

Comparison of equation (A-14) with equation (A-13) gives

$$
\xi=\frac{1}{\left[1+\left[\frac{\gamma-1}{\gamma}\right] \frac{\mathrm{J}_{2}\left[\sqrt{P_{r}} \alpha\right]}{\mathrm{J}_{0}\left[\sqrt{P}_{r} \alpha\right]}\right]}
$$

Equation (A-15) is the same expression as derived by Bergh using a different approach (ref. 6). Equations (A-15) and (A-10) and the equation of state for an ideal gas are used to replace the energy equation throughout the remainder of this analysis.

\section{Momentum Analysis}

The Navier-Stokes momentum equation expressed in cylindrical coordinates is (ref. 11)

$$
\begin{aligned}
& \rho_{0} \frac{\partial U(x, r)}{\partial t}+\frac{\partial P}{\partial x} \\
& =\mu\left[\frac{1}{r} \frac{\partial}{\partial r}\left(r \frac{\partial U(x, r)}{\partial r}\right)+\frac{4}{3} \frac{\partial^{2} U}{\partial x^{2}}\right]
\end{aligned}
$$

Using the equation of continuity for tube flow, linearized for small disturbances

$$
\frac{\partial \rho}{\partial t}+\rho_{0} \frac{\partial U}{\partial x}=0
$$

and the polytropic equation (A-13),

$$
\frac{\partial P}{\partial t}=\frac{\partial P}{\partial \rho} \frac{\partial \rho}{\partial t}=-\frac{\xi}{\gamma} c^{2}\left[\rho_{0} \frac{\partial U}{\partial x}\right]
$$

to eliminate $\partial^{2} U / \partial x^{2}$, and taking the Fourier transform

$$
\begin{aligned}
& \mathrm{j} \omega \rho_{0} U(x, r)+\frac{\partial P}{\partial x} \\
& =\mu\left[\frac{1}{r} \frac{\partial}{\partial r}\left(r \frac{\partial U x, r}{\partial r}\right)-\frac{4}{3} \frac{j \omega}{\rho_{0}} \frac{\partial}{\partial x}\left\{\frac{P}{\xi / \gamma c^{2}}\right\}\right]
\end{aligned}
$$




\section{Collecting terms gives}

$$
\begin{aligned}
U(x, r) & -\frac{\mu}{\mathrm{j} \omega \rho_{0}}\left[\frac{1}{r} \frac{\partial}{\partial r}\left(r \frac{\partial U(x, r)}{\partial r}\right)\right] \\
& =-\frac{1 \partial}{\mathrm{j} \omega \rho_{0} \partial x}\left[\left(1+\frac{4}{3} \frac{\mu}{\rho_{0}}\left\{\frac{\mathrm{j} \omega}{(\xi / \gamma) c^{2}}\right\}\right) P\right]
\end{aligned}
$$

In addition,

$$
\rho_{0}(\xi / \gamma) c^{2}=\xi \rho_{0} R_{g} T \approx \xi \bar{P}_{0}
$$

where $\bar{P}_{0}$ is the mean steady-state pressure at the local temperature. Equation (A-20) becomes

$$
\begin{gathered}
U(x, r)-\frac{\mu}{j \omega \rho_{0}}\left[\frac{1}{r} \frac{\partial}{\partial r}\left(r \frac{\partial U(x, r)}{\partial r}\right)\right] \\
=-\frac{\kappa_{p} \partial P}{j \omega \rho_{0} \partial x}
\end{gathered}
$$

where $\kappa_{p}=\left(1+\frac{4}{3} \frac{j \omega \mu}{\xi P_{0}}\right)$ is a rarefied flow correction term and for air is very close to unity except for rarefied flow conditions. Using a solution method similar to the energy analysis performed earlier, equation (A-22) is integrated with respect to the radial coordinate to give (ref. 6)

$$
U(x, r)=\mathrm{N}(x) \mathrm{J}_{0}\left[\alpha \frac{r}{R}\right]-\frac{\kappa_{p} \frac{\partial P}{\partial x}}{j \omega \rho_{0}}
$$

The constant of integration, $\mathrm{N}(x)$, is solved for using the boundary condition at the wall. For slip flow conditions, the fluid velocity at the wall can be decomposed into two parts: slip velocity and thermomolecular creep velocity. Slip velocity is proportional to the shear stress at the wall. The creep flow is proportional to the longitudinal temperature gradient at the wall. For laminar flow, the wall boundary condition is (ref. 1)

$$
\begin{aligned}
U(x, R) & =U_{\text {slip }}+U_{\text {creep }} \\
& \approx-\vartheta \frac{\partial U}{\partial r}+\frac{3}{4} \frac{\mu_{0} R_{g}}{\widetilde{P}_{0}} \partial T_{w} / \partial x
\end{aligned}
$$

The parameter, $\vartheta$, is referred to as the slip distance. For a given material, this distance can be determined by referring to empirical charts. The parameter $\mu_{0}$ is the local steady-state bulk viscosity in the tubing. Applying equation (A-23) to equation (A-22), solving for the parameter $\mathrm{N}(x)$, and simplifying the solution for slip flow can be written as

$$
\begin{aligned}
U(x, r)= & {\left[\frac{\mathrm{J}_{0}\left[\alpha \frac{r}{R}\right]}{\mathrm{J}_{0}\left[\alpha \frac{r}{R}\right]-\vartheta \frac{\alpha}{R} \mathrm{~J}_{1}\left[\alpha \frac{r}{R}\right]}\right]-1 \frac{\kappa_{p} \frac{\partial P}{\partial x}}{\mathrm{j} \omega \rho_{0}} } \\
& +\left[\frac{\mathrm{J}_{0}\left[\alpha \frac{r}{R}\right]}{\mathrm{J}_{0}\left[\alpha \frac{r}{R}\right]-\vartheta \frac{\alpha}{R} \mathrm{~J}_{1}\left[\alpha \frac{r}{R}\right]}\right] U_{\text {creep }}
\end{aligned}
$$

where $\mathrm{J}_{1}$ is the first-order Bessel function. Averaging over the cross-section of the tube gives

$$
\begin{aligned}
U_{a v g}(x)= & \frac{1}{\pi R^{2}} \int_{0}^{2 \pi} \int_{0}^{R} U(x, r) r d r d \theta \\
= & \frac{\left(\mathrm{J}_{2}[\alpha]+\vartheta \frac{\alpha}{R} \mathrm{~J}_{1}[\alpha]\right)}{\mathrm{J}_{0}[\alpha]-\vartheta \frac{\alpha}{R} \mathrm{~J}_{1}[\alpha]} \frac{\kappa_{p} \frac{\partial P}{\partial x}}{\mathrm{j} \omega \rho_{0}} \\
& +\frac{3 \mu R_{g}}{4 \bar{P}_{0}} \frac{\frac{2}{\alpha} \mathrm{J}_{1}[\alpha]}{\mathrm{J}_{0}[\alpha]-\vartheta \frac{\alpha}{R} \mathrm{~J}_{1}[\alpha]} \frac{\partial T_{w}}{\partial x}
\end{aligned}
$$

Because no radial pressure gradients occur, the polytropic process equation is used to give

$$
\frac{\partial T_{w}}{\partial x}=\frac{\frac{\partial P}{\partial x}}{\frac{\partial P}{\partial T}}=\frac{\xi-1}{\xi} \frac{T}{P} \frac{\partial P}{\partial x}
$$

In addition,

$U_{\text {avg }}$

$\approx \frac{\left\{\kappa_{p}\left(\mathrm{~J}_{2}[\alpha]+\vartheta \frac{\alpha}{R} \mathrm{~J}_{1}[\alpha]\right)+\mathrm{j} \omega \frac{3}{4} \frac{\mu_{0}}{\bar{P}_{0}} \frac{\xi-1}{\xi} \frac{2}{\alpha} \mathrm{J}_{1}[\alpha]\right\}}{\mathrm{J}_{0}[\alpha]-\vartheta \frac{\alpha}{R} \mathrm{~J}_{1}[\alpha]}$

$$
\times \frac{\frac{\partial P}{\partial x}}{j \omega \rho_{0}}
$$


Defining a propagation factor

$\Gamma_{p}$

$$
\equiv \sqrt{\frac{\gamma}{\xi} \frac{\mathrm{J}_{0}[\alpha]-\vartheta \frac{\alpha}{R} \mathrm{~J}_{1}[\alpha]}{\left\{\kappa_{p}\left(\mathrm{~J}_{2}[\alpha]+\vartheta \frac{\alpha}{R} \mathrm{~J}_{1}[\alpha]\right)+\mathrm{j} \omega \frac{3}{4} \frac{\mu_{0}}{\bar{P}_{0}} \frac{\xi-1}{\xi} \frac{2}{\alpha} \mathrm{J}_{1}[\alpha]\right\}}}
$$

the momentum equation for slip flow conditions can finally be written as

$$
\frac{\partial P}{\partial x}=\mathrm{j} \omega \frac{\xi}{\gamma} \Gamma_{p}^{2} \rho_{0} U_{a v g}(x)
$$

\section{Continuity Analysis}

Based on assumptions in eq. (A-1), the radially averaged continuity equation is

$$
\frac{\partial \rho}{\partial t}=\frac{\frac{\partial P}{\partial t}}{\frac{\partial P}{\partial \rho}}=\frac{\gamma}{\xi} \frac{\partial P}{\partial t} \frac{1}{c^{2}}=-\rho_{0}\left(\frac{\partial U_{a v g}}{\partial x}\right)
$$

In addition, the Fourier transform of equation $(\mathrm{A}-31)$ is

$$
\mathrm{j} \omega P=-\left[\frac{\xi}{\gamma} c^{2}\right] \rho_{0}\left(\frac{\partial U_{\text {avg }}}{\partial x}\right)=0
$$

Differentiating equation (A-32) with respect to $x$, and substituting into equation (A-31)

$$
\frac{\partial^{2} P(x)}{\partial x^{2}}=\left[\omega \frac{\Gamma p}{c}\right]^{2} P(x)
$$

Equation (A-33) is the final form of the wave equation which describes the slip flow propagation of pressure waves in the tube.

\section{Evaluation of the Downstream Boundary Condition}

At the downstream end of the tube where the pressure wave exits the pressure tubing and enters the transducer volume, the equation of momentum (eq. (A-32)) still holds, but the equation of continuity must be modified. Here, the integral form of the equation is used and

$$
U_{\text {avg }}(L)=\frac{j \omega \rho V}{\rho_{0} A_{c}}=\frac{\frac{j \omega P V}{\rho_{0} A_{c}}}{\left[\frac{\xi}{\gamma} c^{2}\right]}
$$

where $V$ is the entrapped transducer volume, and $A_{c}$ is the cross-sectional area of the tube at the exit to the transducer. Substituting equation (A-34) into equation (A-32), the downstream boundary condition becomes

$$
\left.\frac{\partial P}{\partial x}\right|_{x=L}=-\frac{\omega^{2} \Gamma_{p}^{2}}{c^{2}} \frac{V}{A_{c}} P_{L}
$$

Equations (A-27), (A-28), (A-33), and (A-35) are the collected boundary value equations.

\section{APPENDIX B SOLUTION OF THE BOUNDARY VALUE EQUATIONS}

As derived in appendix A, the pressure wave propagation equations are

$$
\frac{\partial^{2} P(x)}{\partial x^{2}}=\left[\omega \frac{\Gamma p}{c}\right]^{2} P(x)
$$

The downstream boundary condition is

$$
\frac{\partial P}{\left.\partial x\right|_{x=L}}=-\frac{\omega^{2} \Gamma_{p}^{2}}{c^{2}} \frac{V}{A_{c}} P_{L}
$$

Pressure at the upstream boundary is prescribed. Because several parameters of the boundary value equations vary as a function of the longitudinal temperature distribution in the tube, the equations generally cannot be integrated outright. Conceptually, the set of boundary value equations can be integrated by formulating the problems as a finite difference solution. Unfortunately, the wave equation is hyperbolic, and the downstream boundary condition is parabolic (ref. 12). This mis-match of equation types makes the problem illconditioned for finite difference methods. Depending on the ratio of the time step to the distance step, various degrees of artificial damping will be introduced into the system by a finite difference formulation. This artificial damping makes extracting the true physics from numerical artifacts extremely difficult. 
Instead, a better approach is to integrate the boundary value equations with respect to $x$, assuming that the gas properties remain constant along the region of integration. This longitudinally averaged model can be solved in closed form in the frequency domain to give a fundamental solution. Using this fundamental solution as a building block, the problem can be solved recursively starting at the transducer end and working toward the surface end of the tube. If the properties of the flow are re-evaluated at each new node, then one may effectively allow for the construction of a solution in which the flow properties are arbitrarily variable as a function of $x$. This finite-element approach is an integral method that is not subject to the numerical problems encountered with the finite difference methods.

\section{Fundamental Solution of the Boundary Value Equations}

Across the tube, assume constant flow properties. Integrating equation (B-1) with respect to $x$ gives

$$
\begin{aligned}
P(x, \omega)= & \mathrm{A}_{\omega} \mathrm{e}\left(\omega \Gamma_{p} \frac{x}{c}\right) \\
& +\mathrm{B}_{\omega} \mathrm{e}\left(-\omega \Gamma_{p} \frac{x}{c}\right)
\end{aligned}
$$

Applying the upstream and downstream boundary conditions to solve for $A_{\omega}$ and $B_{\omega}$, substituting the results into equation (B-3), and simplifying gives

$P(x, \omega)$

$$
=P_{0}(\omega)\left[\frac{\cosh \left[\omega \Gamma_{p} \frac{x-L}{c}\right]-\frac{V \sqrt{\alpha}}{A_{c} \bar{c}} \sinh \left[\omega \Gamma_{p} \frac{x-L}{c}\right]}{\cosh \left[\omega \Gamma_{p} \frac{L}{c}\right]+\frac{V \omega \Gamma_{p}}{A_{c} c} \sinh \left[\omega \Gamma_{p} \frac{L}{c}\right]}\right]
$$

Evaluating equation (B-4) at $x=L$ gives the end-toend solution:

$P_{L}(\omega)$

$$
\left.=P_{0}(\omega)\left[\frac{1}{\cosh \left[\omega \Gamma_{p}\right.} \frac{\left.\frac{L}{c}\right]+\frac{V \omega \Gamma_{p}}{A_{c} c} \sinh \left[\omega \Gamma_{p}\right.}{\frac{L}{c}}\right]\right]
$$

Equation (B-5) is a frequency response model where the complex spectra are given as a function of the sensor geometry, the frequency of the input sinusoid, and the propagation factor, $\Gamma_{p}$. Molecular effects are all embedded in $\Gamma_{p}$. If molecular effects are ignored, equations (B-4) and (B-5) are identical to the fundamental solutions developed by Bergh, et al., (refs. 6, 7).

\section{Recursive Solution of the Boundary Value Equations}

The fundamental solution is limited to applications where the temperature gradients are small, and the tube radius is constant. However by using equations (B-4) and (B-5) as building blocks, solutions allowing longitudinal variation of the fluid properties and complex tube geometries can be constructed. The solution is recursive and moves from the downstream boundary to the upstream boundary. The solution is performed assuming $\mathrm{n}$ solution elements. These elements are not necessarily evenly spaced. The junctions of elements are referred to as nodes. Within each element, the fluid properties are assumed to be constant, but properties between nodes are allowed to vary. At each node, the equation of continuity is satisfied, giving a new downstream condition. For generality, the tube radius is allowed to vary, and a volume is assumed to be entrapped at each node. These entrapped volumes can be used to model the effects of tube joints, fittings, or other devices, such as water traps.

First, consider a two-node system with node $\mathrm{n}$ being the transducer node and node $n-1$ being the adjacent upstream node. The configuration being analyzed is depicted in figure A-1. At node $\mathrm{n}-1$, the integral form of the continuity equation is

$$
\begin{aligned}
U_{\mathrm{n}-1}= & \frac{j \omega}{\rho_{0}}\left\{\frac{V_{\mathrm{n}-1}}{A_{c_{\mathrm{n}-1}}} \frac{P_{\mathrm{n}-1}}{\xi / \gamma c_{\mathrm{n}-1}^{2}}\right\} \\
& +\frac{A_{c_{\mathrm{n}}}}{A_{c_{\mathrm{n}-1}}} \int_{L_{\mathrm{N}-1}}^{L_{\mathrm{N}}} P /\left(\frac{\xi}{\gamma} c_{x, \omega^{2}}\right) d x \\
& +\frac{V_{\mathrm{n}}}{A_{c_{\mathrm{n}-1}}} \frac{P_{\mathrm{n}}}{(\xi / \gamma) c_{\mathrm{n}}^{2}}
\end{aligned}
$$

Substituting the downstream boundary condition (eq. (B-2)) for $U_{\mathrm{n}-1}$ yields 


$$
\begin{aligned}
\frac{\partial P(\mathrm{n}-1, \omega)}{\partial x}= & -\frac{V_{\mathrm{n}-1}}{A_{c_{\mathrm{n}-1}}} \frac{\omega^{2} \Gamma_{p_{\mathrm{n}-1}}^{2}}{c_{\mathrm{n}-1}^{2}}\left\{P_{\mathrm{n}-1}\right. \\
& +\frac{A_{c_{\mathrm{n}}}}{V_{\mathrm{n}-1}} \frac{c_{\mathrm{n}-1}^{2}}{c_{\mathrm{n}}^{2}} \int_{L_{\mathrm{n}-1}}^{L_{\mathrm{n}}} P d x \\
& \left.+\frac{c_{\mathrm{n}-1}^{2}}{c_{\mathrm{n}}^{2}} \frac{V_{\mathrm{n}}}{V_{\mathrm{n}-1}} P_{\mathrm{n}}\right\}
\end{aligned}
$$

To evaluate the integral in equation (B-7), the fundamental solution (eq. (B-4)) is used. The fundamental solution is valid from node $n-1$ to node $n$ because the downstream boundary condition is identical to that of the fundamental solution. Performing the integration, simplifying, and collecting terms equation (B-7) reduces to

$$
\frac{\partial P(\mathrm{n}-1)}{\partial x}=-\frac{V_{e_{\mathrm{n}-1}}}{A_{c_{\mathrm{n}-1}}} \frac{\omega^{2} \Gamma_{p_{\mathrm{n}-1}}^{2}}{c_{\mathrm{n}-1}{ }^{2}} p_{\mathrm{n}-1}
$$

where $V_{e}$ is the effective volume parameter which accounts for the impedance of the downstream tube and volume as well as the volume at node $n-1$.

$$
\begin{aligned}
V_{e_{\mathrm{n}-1}}= & V_{\mathrm{n}-1} \\
& +\frac{c_{\mathrm{n}-1}^{2} V_{\mathrm{n}}}{c_{\mathrm{n}}^{2}}\left[\cosh \left[\omega \Gamma_{p_{\mathrm{n}}} \frac{L_{\mathrm{n}}}{c_{\mathrm{n}}}\right]\right. \\
& +\frac{1}{\omega \frac{V_{\mathrm{n}}}{A_{c_{\mathrm{n}}}} \frac{\Gamma_{p_{\mathrm{n}}}}{c_{\mathrm{n}}}} \sinh \left[\omega \Gamma_{p_{\mathrm{n}}} \frac{L_{\mathrm{n}}}{c_{\mathrm{n}}}\right] \\
& +\left(\cosh \left[\omega \Gamma_{p_{\mathrm{n}}} \frac{L_{\mathrm{n}}}{c_{\mathrm{n}}}\right]\right. \\
& \left.+\omega \frac{V_{\mathrm{n}}}{A_{c_{\mathrm{n}}}} \frac{\Gamma_{p_{\mathrm{n}}}}{c_{\mathrm{n}}} \sinh \left[\omega \Gamma_{p_{\mathrm{n}}} \frac{L_{\mathrm{n}}}{c_{\mathrm{n}}}\right]\right)
\end{aligned}
$$

The form of the new boundary condition given by equation (B-8) is identical to the original boundary condition at node $\mathrm{n}$. By induction on the fundamental solution (at node $n$ ), the solution at node $n-1$ is

$$
\begin{aligned}
P_{\mathrm{n}-1}(\omega)= & \left(P_{\mathrm{n}-2}(\omega)\right) \\
& +\left(\cosh \left[\omega \Gamma_{p_{\mathrm{n}-1}} \frac{L_{\mathrm{n}-1}}{c_{\mathrm{n}-1}}\right]\right. \\
& \left.+\omega \frac{V_{e_{\mathrm{n}-1}}}{A_{c_{\mathrm{n}-1}}} \frac{\Gamma_{p_{\mathrm{n}-1}}}{c_{\mathrm{n}-1}} \sinh \left[\omega \Gamma_{p_{\mathrm{n}-1}} \frac{L_{\mathrm{n}-1}}{c_{\mathrm{n}-1}}\right]\right)
\end{aligned}
$$

To establish generality, the process must be repeated an additional time. Repeating the process at node $n-2$, the downstream boundary condition is

$$
\begin{aligned}
\frac{\partial P_{\mathrm{n}-2}}{\partial x}= & -\omega \frac{V_{\mathrm{n}-2} \Gamma_{p_{\mathrm{n}-2}}}{A_{c_{\mathrm{n}-2}} c_{n-2}^{2}}\left\{P_{\mathrm{n}-2}\right. \\
& +\frac{A_{c_{\mathrm{n}-1}} c_{\mathrm{n}-2}^{2}}{V_{\mathrm{n}-2} c_{\mathrm{n}-1}^{2}} \int_{L_{\mathrm{n}-2}}^{L_{\mathrm{n}-1}} P d x \\
& +\frac{c_{\mathrm{n}-2}^{2} V_{\mathrm{n}-1}}{c_{\mathrm{n}-1}^{2} V_{\mathrm{n}-2}}\left\{P_{\mathrm{n}-1}\right. \\
& +\frac{c_{\mathrm{n}-1}^{2} A_{c_{\mathrm{n}}}}{c_{\mathrm{n}}^{2} V_{\mathrm{n}-1}} \int_{L_{\mathrm{n}-1}}^{L_{\mathrm{n}}} P d x \\
& \left.+\frac{\left.\left.V_{\mathrm{n}} \frac{c_{\mathrm{n}-1}^{2}}{V_{\mathrm{n}-1}} P_{\mathrm{n}}\right\}\right\}}{c_{\mathrm{n}}}\right\}
\end{aligned}
$$

However, from the solution at node $\mathrm{n}-1$,

$$
\begin{aligned}
\left\{P_{\mathrm{n}-1}\right. & +\frac{A_{c_{\mathrm{n}}} c_{\mathrm{n}-1}{ }^{2}}{V_{\mathrm{n}-1} c_{\mathrm{n}}^{2}} \int_{L_{\mathrm{n}-1}}^{L_{\mathrm{n}}} P d x \\
+ & \left.\frac{V_{\mathrm{n}}}{V_{\mathrm{n}-1}} \frac{c_{\mathrm{n}-1}^{2}}{c_{\mathrm{n}}^{2}} P_{\mathrm{n}}\right\}=\frac{V_{e_{\mathrm{n}-1}}}{V_{\mathrm{n}-1}} P_{\mathrm{n}-1}
\end{aligned}
$$


In addition, equation ( $\mathrm{B}-12)$ becomes the new boundary condition at node $\mathrm{n}-2$.

$$
\begin{aligned}
\frac{\partial P_{\mathrm{n}-2}}{\partial x}= & -\omega \frac{V_{\mathrm{n}-2} \Gamma_{p_{\mathrm{n}-2}}}{A_{c_{\mathrm{n}-2}} c_{\mathrm{n}-2}^{2}}\left\{P_{\mathrm{n}-2}\right. \\
& +\frac{A_{c_{\mathrm{n}-1}} c_{\mathrm{n}-2}}{V_{\mathrm{n}-2} c_{\mathrm{n}-1}{ }^{2}} \int_{L_{\mathrm{n}-2}}^{L_{\mathrm{n}-1}} P d x \\
& \left.+\frac{c_{\mathrm{n}-2}^{2} V_{e_{\mathrm{n}-1}}}{c_{\mathrm{n}-1}^{2} V_{\mathrm{n}-2}} P_{\mathrm{n}-1}\right\}
\end{aligned}
$$

This form of equation (B-13) is identical to the boundary condition at node n-1 (eq. (B-7)). Again by induction on the solution at node $n-1$, the solution at node $\mathrm{n}-2$ can be written as

$$
\begin{aligned}
P_{\mathrm{n}-2}(\omega)= & \left(P_{\mathrm{n}-3}(\omega)\right) \\
& +\left(\cosh \left[\omega \Gamma_{p_{\mathrm{n}-2}} \frac{L_{\mathrm{n}-2}}{c_{\mathrm{n}-2}}\right]\right. \\
& \left.+\omega \frac{V_{e_{\mathrm{n}-2}}}{A_{c_{\mathrm{n}-2}}} \frac{\Gamma_{p_{\mathrm{n}-2}}}{c_{\mathrm{n}-2}} \sinh \left[\omega \Gamma_{p_{\mathrm{n}-2}} \frac{L_{\mathrm{n}-2}}{c_{\mathrm{n}-2}}\right]\right)
\end{aligned}
$$

where the effective volume at node $n-2$ is

$$
\begin{aligned}
V_{e_{\mathrm{n}-2}}= & V_{\mathrm{n}-2} \\
& +\frac{c_{\mathrm{n}-1}^{2} V_{e_{\mathrm{n}-1}}}{c_{\mathrm{n}-1}^{2}}\left[\cosh \left[\omega \Gamma_{p_{\mathrm{n}-1}} \frac{L_{\mathrm{n}-1}}{c_{\mathrm{n}-1}}\right]\right. \\
& \left.+\frac{1}{\omega \frac{V_{e_{\mathrm{n}-1}}}{A_{c_{\mathrm{n}-1}}} \frac{\Gamma_{p_{\mathrm{n}-1}}}{c_{\mathrm{n}-1}}} \sinh \left[\omega \Gamma_{p_{\mathrm{n}-1}} \frac{L_{\mathrm{n}-1}}{c_{\mathrm{n}-1}}\right]\right] \\
& +\left(\cosh \left[\omega \Gamma_{p_{\mathrm{n}-1}} \frac{L_{\mathrm{n}-1}}{c_{\mathrm{n}-1}}\right]\right. \\
& +\omega \frac{\left.V_{e_{\mathrm{n}-1}} \frac{\Gamma_{p_{\mathrm{n}-1}}}{A_{c_{\mathrm{n}-1}}} \sinh \left[\omega \Gamma_{p_{\mathrm{n}-1}} \frac{L_{\mathrm{n}-1}}{c_{\mathrm{n}-1}}\right]\right)}{}
\end{aligned}
$$

\section{General Solution Using}

Recursive Formulation

The general end-to-end frequency response is given as the complex product of the frequency responses at the individual nodes. By induction on the previous solutions, the solution at the $i t h$ node is

$$
\begin{aligned}
& P_{\mathrm{i}}(\omega) \\
& =\frac{P_{\mathrm{i}-1}(\omega)}{\cosh \left[\omega \Gamma_{p_{\mathrm{i}}} \frac{L_{\mathrm{i}}}{c_{\mathrm{i}}}\right]+\omega \frac{V_{e_{\mathrm{i}}}}{A_{c_{\mathrm{i}}}} \frac{\Gamma_{p_{\mathrm{i}}}}{c_{\mathrm{i}}} \sinh \left[\omega \Gamma_{p_{\mathrm{i}}} \frac{L_{\mathrm{i}}}{c_{\mathrm{i}}}\right]}
\end{aligned}
$$

where the effective volume is

$$
V_{e_{\mathrm{i}}}=V_{\mathrm{i}}
$$

$$
\begin{aligned}
& +\frac{c_{\mathrm{i}}^{2} V_{e_{\mathrm{i}+1}}}{c_{\mathrm{i}}^{2}}\left[\cosh \left[\omega \Gamma_{p_{\mathrm{i}+1}} \frac{L_{\mathrm{i}+1}}{c_{\mathrm{i}+1}}\right]\right. \\
& \left.+\frac{1}{\omega \frac{V_{e_{\mathrm{i}+1}}}{A_{c_{\mathrm{i}+1}}} \frac{\Gamma_{p_{\mathrm{i}+1}}}{c_{\mathrm{i}+1}}} \sinh \left[\omega \Gamma_{p_{\mathrm{i}+1}} \frac{L_{\mathrm{i}+1}}{c_{\mathrm{i}+1}}\right]\right]
\end{aligned}
$$

$$
\begin{aligned}
& +\left(\cosh \left[\omega \Gamma_{p_{\mathrm{i}+1}} \frac{L_{\mathrm{i}+1}}{c_{\mathrm{i}+1}}\right]\right. \\
& \left.+\omega \frac{V_{e_{\mathrm{i}+1}}}{A_{c_{\mathrm{i}+1}}} \frac{\Gamma_{p_{\mathrm{i}+1}}}{c_{\mathrm{i}+1}} \sinh \left[\omega \Gamma_{p_{\mathrm{i}+1}} \frac{L_{\mathrm{i}+1}}{c_{\mathrm{i}+1}}\right]\right)
\end{aligned}
$$

with $V_{e_{\mathrm{n}}}=V_{\mathrm{n}}$. In addition,

$$
\begin{aligned}
& \frac{P_{L}(\omega)}{P_{0}(\omega)} \\
& =\frac{P_{1}(\omega)}{P_{0}(\omega)} \frac{P_{2}(\omega)}{P_{1}(\omega)} \cdots \frac{P_{\mathrm{n}-1}(\omega)}{P_{\mathrm{n}-2}(\omega)} \frac{P_{\mathrm{n}}(\omega)}{P_{\mathrm{n}-1}(\omega)} \\
& =\prod_{\mathrm{i}=1}^{\mathrm{n}} \frac{1}{\cosh \left[\omega \Gamma_{p_{\mathrm{i}}} \frac{L_{\mathrm{i}}}{c_{\mathrm{i}}}\right]+\omega \frac{V_{e_{\mathrm{i}}}}{A_{c_{\mathrm{i}}}} \frac{\Gamma_{p_{\mathrm{i}}}}{c_{\mathrm{i}}} \sinh \left[\omega \Gamma_{p_{\mathrm{i}}} \frac{L_{\mathrm{i}}}{c_{\mathrm{i}}}\right]}
\end{aligned}
$$


Equations (B-17) and (B-18) represent a finiteelement solution of the boundary value problem. The boundary value equations have been analytically integrated at each element based on simplifying assumptions. The method is not subject to the numerical problems encountered with the finite difference methods. The solution assumes that a tube surface temperature profile is prescribed at each node, and the fluid properties, namely the temperature, sonic velocity, and dynamic viscosity, are evaluated as a function of the prescribed temperature using the energy equation (A-10).

\section{APPENDIX C}

\section{STEADY-STATE RESPONSE OF TUBE RESPONSE MODEL FOR RAREFIED FLOW CONDITIONS}

If the general solution (eq. (B-18)) is evaluated at low frequency with large longitudinal temperature gradients and rarefied flow conditions, the gain does not approach 1 as it does for continuum flow conditions. Instead, the hot end of the tube has a higher pressure magnitude than the cold end. This equilibrium pressure gradient is a well-known result. The equilibrium behavior of the model for rarefied flow conditions is best understood by looking at the momentum equation for a cross-section of the pressure tubing.

Evaluating the steady-state behavior of equation (A-28) using the final value theorem yields (ref. 16)

$$
\begin{aligned}
& U_{s s}=\frac{\mathrm{j} \omega}{\lim \omega \Rightarrow 0} \\
& {\left[\frac{\kappa_{p}\left\{\mathrm{~J}_{2}[\alpha]+\vartheta \frac{\alpha}{R} \mathrm{~J}_{1}[\alpha]+\mathrm{j} \omega \frac{3}{4} \frac{\mu_{0}}{\bar{P}_{0}} \frac{\xi-1}{\xi} \frac{2}{\alpha} \mathrm{J}_{1}[\alpha]\right\}}{\mathrm{J}_{0} \alpha-\vartheta \frac{\alpha}{R} \mathrm{~J}_{1} \alpha}\right.} \\
& \left.\times \frac{\frac{\partial P}{\partial x}}{j \omega \rho_{0}}\right]
\end{aligned}
$$

Using the series expansion form for Bessel functions yields

$$
\mathrm{J}_{\mathrm{n}}[\alpha]=\sum_{k=0}^{\infty} \frac{(-1)^{k}}{k !(n+k) !}\left(\frac{\alpha}{2}\right)^{\mathrm{n}+2 k}
$$

Then,

$$
\begin{aligned}
& \frac{\mathrm{J}_{0}[\alpha]}{\lim \omega \Rightarrow 0}=1, \frac{\mathrm{J}_{1}[\alpha]}{\lim \omega \Rightarrow 0}=\frac{\alpha}{2}, \\
& \frac{\mathrm{J}_{2}[\alpha]}{\lim \omega \Rightarrow 0}=\frac{\alpha^{2}}{8}, \kappa_{p}=1
\end{aligned}
$$

In addition,

$$
U_{s s}=\left[\frac{1}{\rho_{0}}\left\{\frac{\alpha^{2}}{8}+\frac{\vartheta}{R} \frac{\alpha^{2}}{2}+j \omega \frac{3}{4} \frac{\mu_{0}}{\bar{P}_{0}} \frac{\xi-1}{\xi}\right\} \frac{\partial P}{\partial x}\right] \text { (C-4) }
$$

but for equilibrium flow conditions, there must be no net flow across any cross-section of the tube, and $U_{s s}=0$. As a result,

$$
\frac{\alpha^{2}}{8}\left(1+4 \frac{\vartheta}{R}\right) \frac{\partial P}{\partial x}=-\mathrm{j} \omega \frac{3}{4} \frac{\mu_{0}}{\bar{P}_{0}} \frac{\xi-1 \partial P}{\xi \partial x}
$$

but $\alpha^{2}=-j \omega \frac{\rho_{0} R^{2}}{\mu_{0}}$. Using the polytropic process energy equation (eq. (A-13)) after some simplification yields

$$
\begin{aligned}
\frac{\frac{\partial P}{\partial x}}{P} & =\frac{6}{\left(1+4 \frac{\vartheta}{R}\right)} \frac{\mu_{0}^{2}}{\rho_{0} R^{2} P_{0}} \frac{\frac{\partial T}{\partial x}}{T} \\
& =\frac{6}{\left(1+4 \frac{\vartheta}{R}\right)} \frac{\mu_{0}^{2} R_{g} T_{0}}{R^{2} \bar{P}_{0}^{2}} \frac{\frac{\partial T}{\partial x}}{T}
\end{aligned}
$$

If the ratio of slip distance to mean free path is defined as $\varepsilon=\vartheta / \lambda$, then

$$
\kappa^{2} \approx \pi R_{g} \frac{\mu^{2}}{R^{2}} \frac{T}{P^{2}}, \frac{\vartheta}{R}=\varepsilon \frac{\lambda}{R}=\varepsilon \kappa
$$

Equation (C-6) becomes

$$
\frac{\partial P}{P} \approx \frac{6 \kappa_{0}^{2}}{\pi\left(1+4 \varepsilon \kappa_{0}\right)} \frac{\frac{\partial T}{\partial x}}{T}
$$


Clearly, based on equation (C-8), the normalized pressure gradient is proportional to the normalized temperature gradient and mean properties of the flow given by $\kappa_{0}$. This equation is used to evaluate the range of Knudsen numbers for which the rarefied flow model is valid.

\section{REFERENCES}

${ }^{1}$ Kennard, Earle H., Kinetic Theory of Gases, McGraw-Hill, New York, 1938, pp. 311-337.

2Iberall, Arthur S., Attenuation of Oscillatory Pressures in Instrument Lines, U.S. National Bureau of Standards Report RP2115, vol. 45, July 1950.

${ }^{3}$ Lamb, J.P., Jr., The Influence of Geometry Parameters Upon Lag Error in Airborne Pressure Measurement Systems, WADC TR 57-351, Wright-Patterson AFB, Ohio, July 1957.

${ }^{4}$ Schuder, C.B. and Binder, R.C., "The Response of Pneumatic Transmission Lines to Step Inputs," Transactions of the American Society of Mechanical Engineers, Dec. 1959.

${ }^{5}$ Hougen, J.O., Martin, O.R., and Walsh, R.A., "Dynamics of Pneumatic Transmission Lines," Journal of Control Engineering, March 1960.

${ }^{6} \mathrm{Bergh}, \mathrm{H}$. and Tijdeman, H., Theoretical and Experimental Results for the Dynamic Response of Pressure Measuring Systems, NLR-TR F.238, National Aero- and Astronautical Research, Amsterdam, Jan. 1965.

${ }^{7}$ Tijdeman, H. and Bergh, H., The Influence of the Main Flow on the Transfer Function of TubeTransducer Systems Used for Unsteady Pressure Measurements, NLR-MP 72023, National Aero- and Astronautical Research, Amsterdam, 1972.
${ }^{8}$ Tijdeman, H., Investigations of the Transonic Flow Around Oscillating Airfoils, NLR-TR 77090, National Aero- and Astronautical Research, Amsterdam, 1977.

${ }^{9}$ Parrot, T. and Zorumski, W., "Sound Transmission Through a High-Temperature Acoustic Probe Tube," AIAA 90-3991, Oct. 1990.

${ }^{10}$ Knudsen, von Martin, "Eine Revision der Gleichgewichtsbedingung der Gase: Thermische Molekularströmung," Annalen der Physik, vol. 31, Nov. 1910, pp. 205-229.

${ }^{11}$ Stephens, R.W.B. and Bate, A.E., Acoustics and Vibrational Physics, St. Martin Press, New York, 1966.

${ }^{12}$ Freiberger, W.F., ed., The International Dictionary of Applied Mathematics, D. Van Nostrand Co., Inc., Princeton, 1960, pp. 17.

${ }^{13}$ Lennart, Rade and Westergren, Bertil, Beta Mathematics Handbook, CRC Press, Boca Raton, 1992.

${ }^{14}$ Bendat, Julius S. and Piersol, Allan G., Random Data: Analysis and Measurement Procedures, Wiley \& Sons, New York, 1971.

${ }^{15}$ Doebelin, Ernest O., Measurement Systems: Application and Design, 2nd ed., McGraw-Hill, New York, 1983, pp. 404-456.

${ }^{16}$ Franklin, Gene F. and Powell, J. David, Digital Control of Dynamic Systems, Addison Wesley, Reading, 1980. 


\section{TABLES}

Table 1. Frequency response test matrix.

\begin{tabular}{cccccrrrr}
\hline \hline & Diameter, & Length, & \multicolumn{6}{c}{ Thermocouple readings along tube, ${ }^{\circ} \mathrm{F}$} \\
\cline { 4 - 9 } Run & in. & in. & 1 & \multicolumn{1}{c}{2} & \multicolumn{1}{c}{3} & \multicolumn{1}{c}{4} & \multicolumn{1}{c}{5} & \multicolumn{1}{c}{6} \\
\hline 1 & 0.066 & 12 & 74.0 & 74.0 & 75.0 & 74.0 & 77.0 & 84.2 \\
2 & 0.066 & 12 & 74.8 & 225.1 & 338.0 & 344.0 & 218.0 & 85.1 \\
3 & 0.066 & 12 & 77.8 & 309.0 & 496.0 & 503.0 & 312.0 & 89.1 \\
4 & 0.066 & 12 & 79.4 & 381.0 & 636.0 & 644.0 & 385.0 & 90.1 \\
5 & 0.066 & 12 & 80.4 & 383.0 & 639.0 & 647.0 & 387.0 & 92.9 \\
6 & 0.066 & 12 & 78.0 & 311.0 & 501.0 & 508.0 & 315.0 & 92.4 \\
7 & 0.066 & 12 & 77.0 & 228.0 & 340.0 & 348.0 & 221.0 & 88.9 \\
8 & 0.066 & 12 & 77.0 & 77.2 & 77.4 & 78.0 & 77.4 & 88.4 \\
9 & 0.033 & 12 & 74.0 & 74.0 & 75.0 & 74.0 & 77.0 & 84.2 \\
10 & 0.033 & 12 & 77.0 & 233.3 & 354.0 & 360.9 & 230.0 & 91.8 \\
11 & 0.033 & 12 & 79.8 & 316.3 & 501.2 & 511.8 & 312.0 & 105.3 \\
12 & 0.033 & 12 & 81.6 & 392.6 & 641.5 & 652.3 & 389.8 & 113.3 \\
13 & 0.033 & 12 & 81.7 & 392.4 & 641.4 & 653.1 & 390.0 & 113.5 \\
14 & 0.033 & 12 & 79.8 & 317.0 & 502.3 & 512.2 & 312.4 & 105.4 \\
15 & 0.033 & 12 & 75.0 & 231.6 & 352.0 & 359.9 & 230.0 & 91.5 \\
16 & 0.033 & 12 & 77.0 & 77.0 & 77.3 & 77.2 & 77.0 & 88.2 \\
\hline \hline
\end{tabular}

Table 2. Rarefied flow condition test matrix.

\begin{tabular}{cccccc}
\hline \hline \multirow{2}{*}{$\begin{array}{c}\text { Diameter, } \\
\text { in. }\end{array}$} & \multicolumn{2}{c}{ Tube temperatures, ${ }^{\circ} \mathrm{F}$} & & \multicolumn{2}{c}{ Pressure, psf } \\
\cline { 2 - 3 } \cline { 5 - 6 } & Hot & Cold & & Minimum & Maximum \\
\hline 0.014 & 499.700 & 75.600 & & 0.28 & 1952.2 \\
0.014 & 950.700 & 75.600 & & 0.56 & 1953.3 \\
0.033 & 499.700 & 75.600 & & 1.68 & 1953.3 \\
0.066 & 433.000 & 81.000 & 1.87 & 1951.2 \\
0.066 & 953.300 & 83.900 & 3.50 & 1949.1 \\
0.080 & 949.800 & 84.200 & 1.69 & 1951.2 \\
0.092 & 431.800 & 82.100 & 1.99 & 1949.6 \\
0.092 & 501.200 & 76.800 & 2.80 & 1951.0 \\
\hline \hline
\end{tabular}




\section{FIGURES}

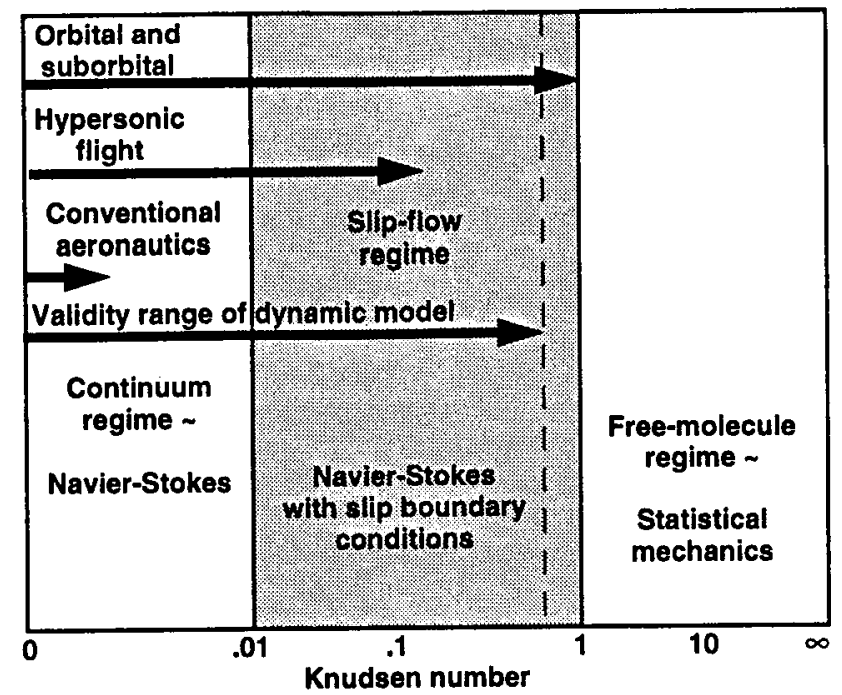

Figure 1. Knudsen number ranges for continuum, slip, and molecular flow regimes.

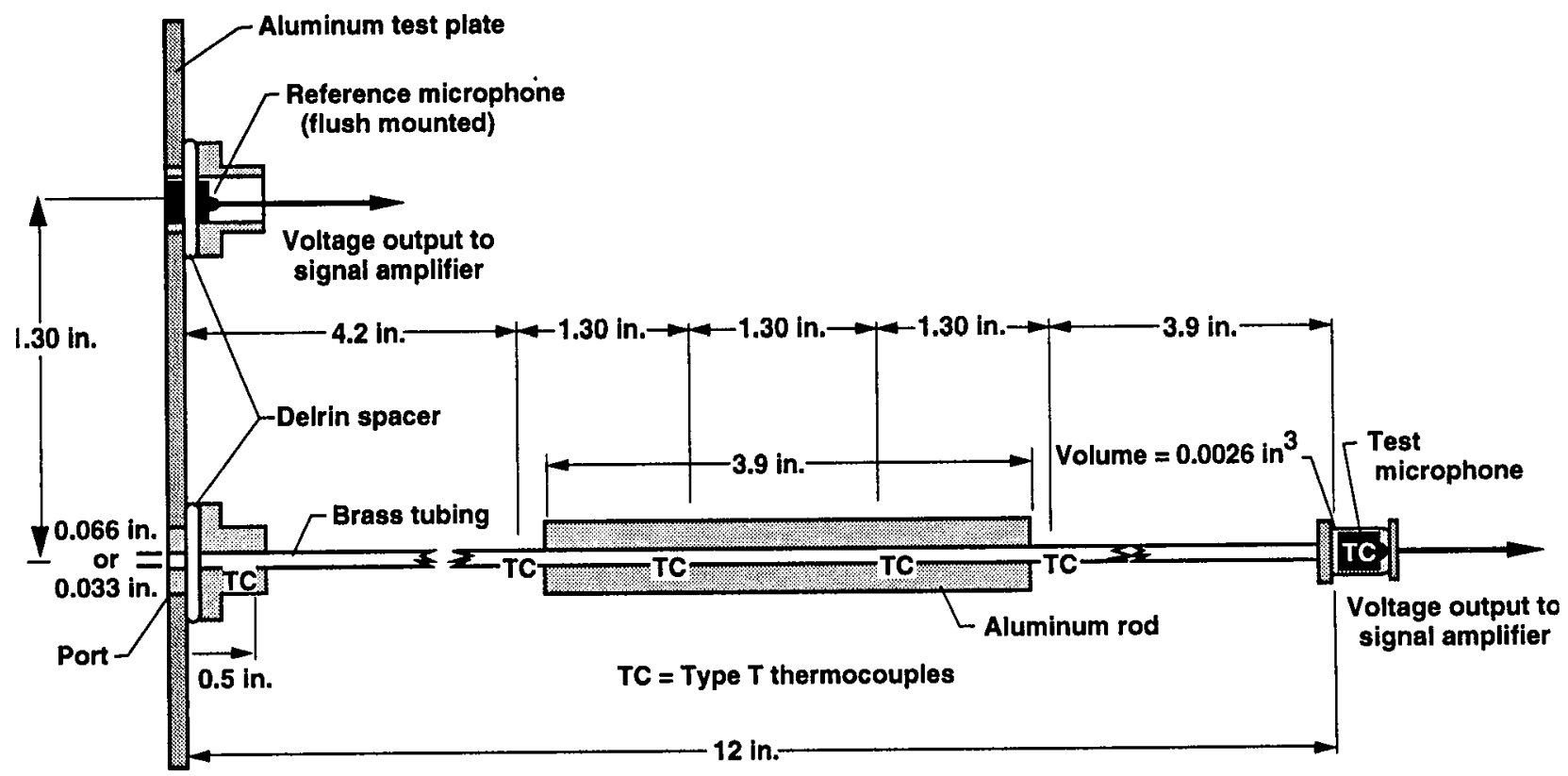

(a) Sensor geometry for heating tests.

Figure 2. Experimental apparatus used for frequency response tests. 


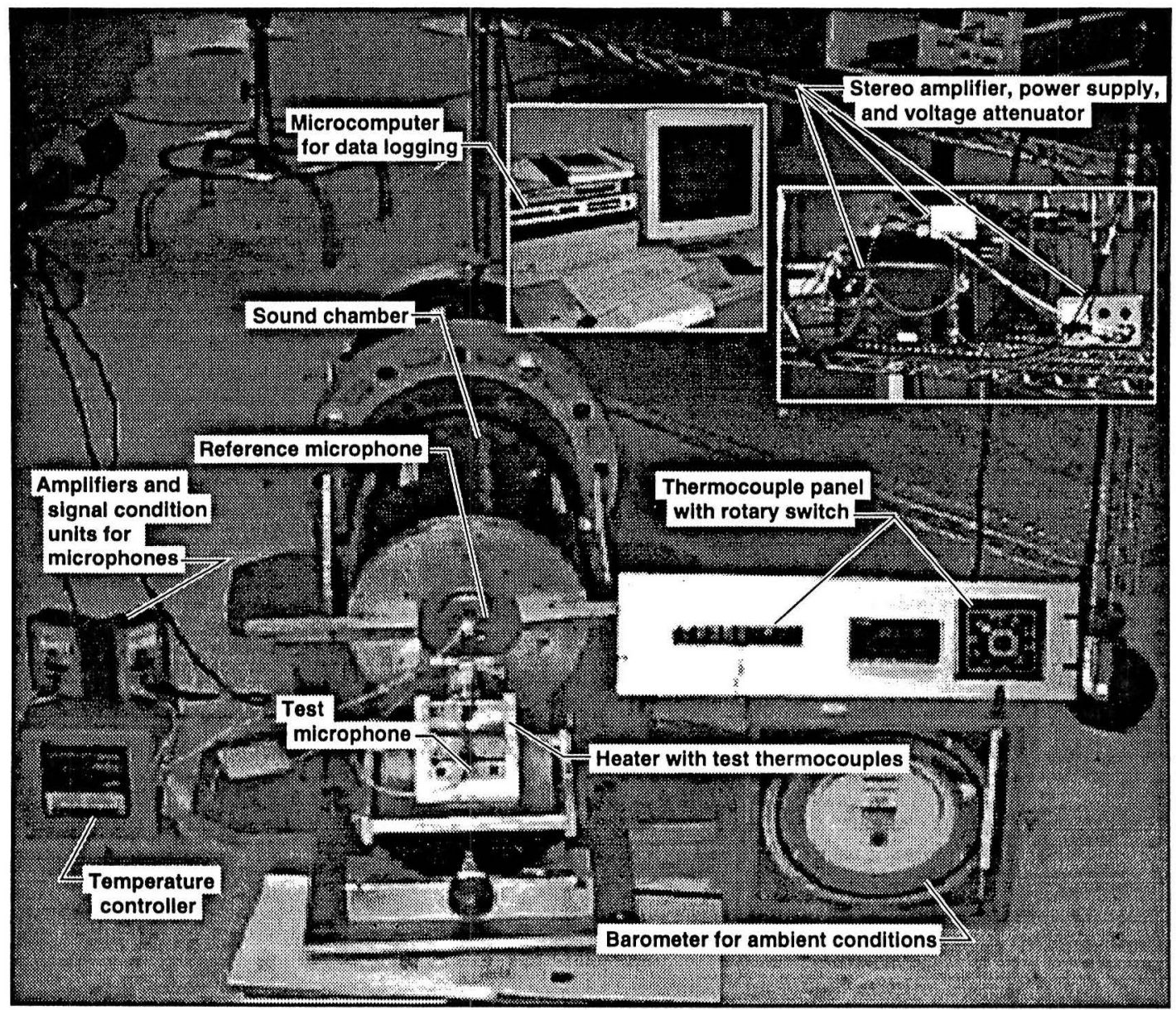

(b) Equipment layout.
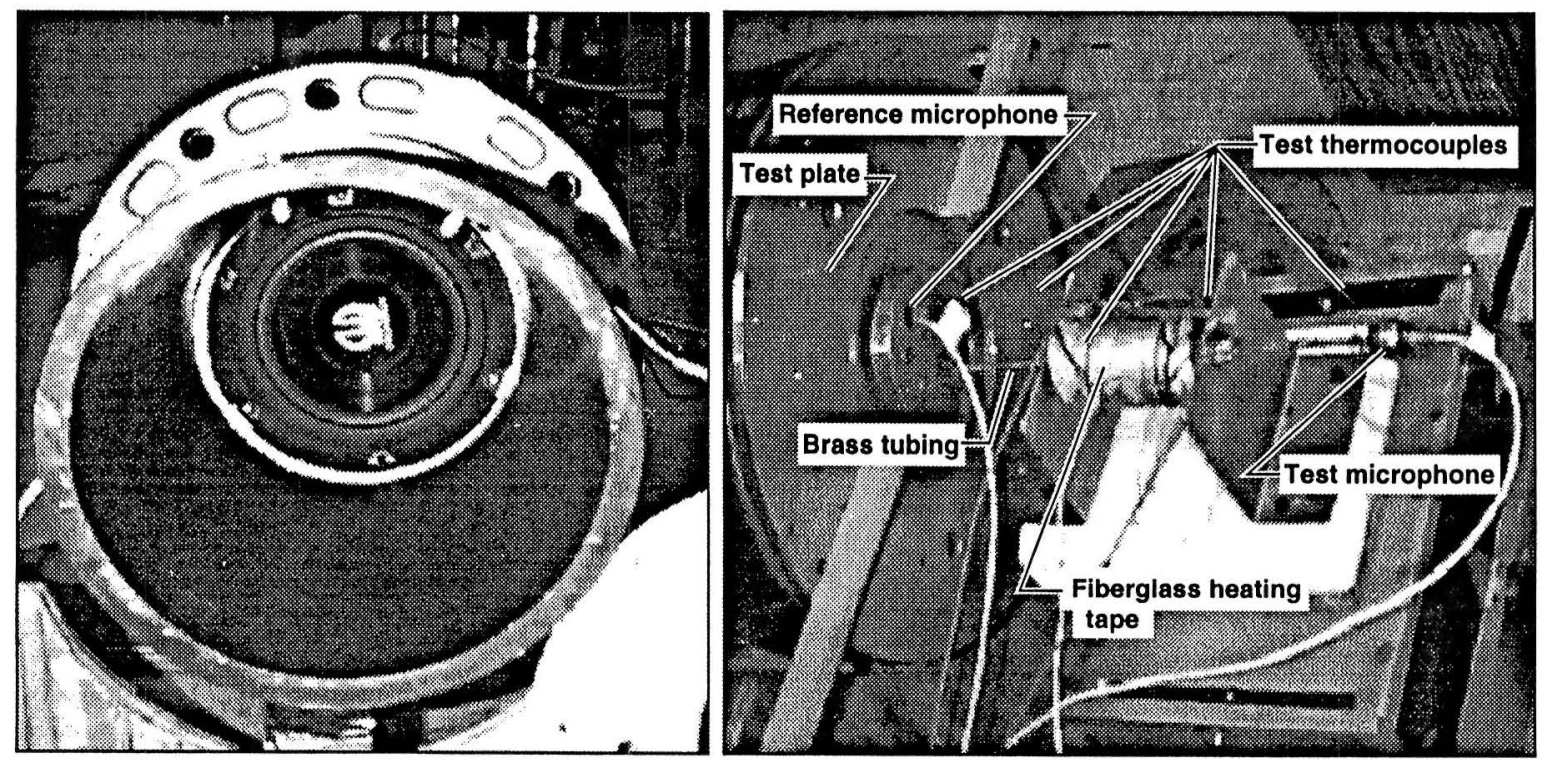

(c) Speaker mounted in sound chamber and the heater apparatus and test plate.

Figure 2. Concluded. 


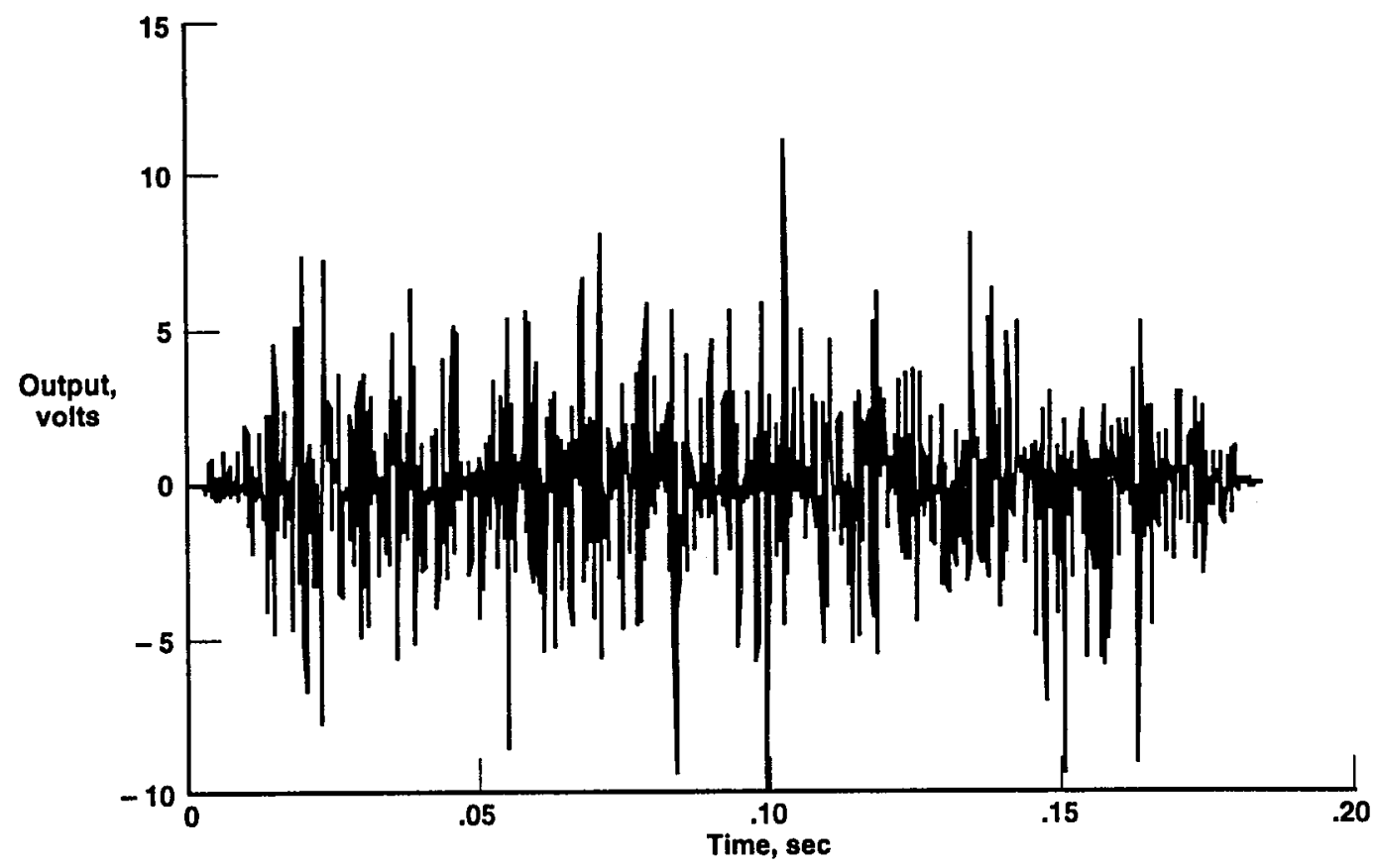

(a) Time history.

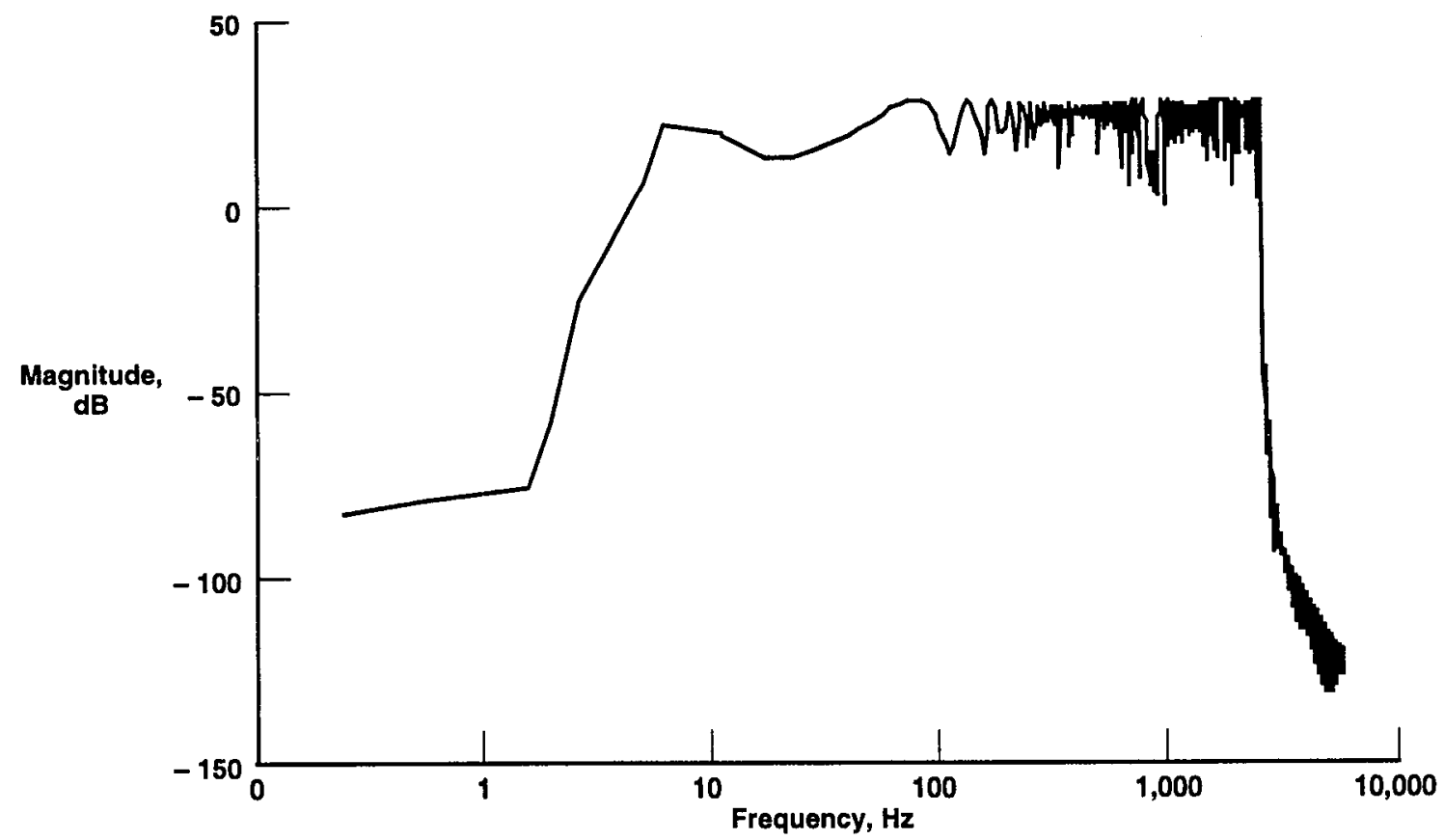

(b) Spectrum.

Figure 3. Typical phase-modulated output waveform used for frequency response tests. 


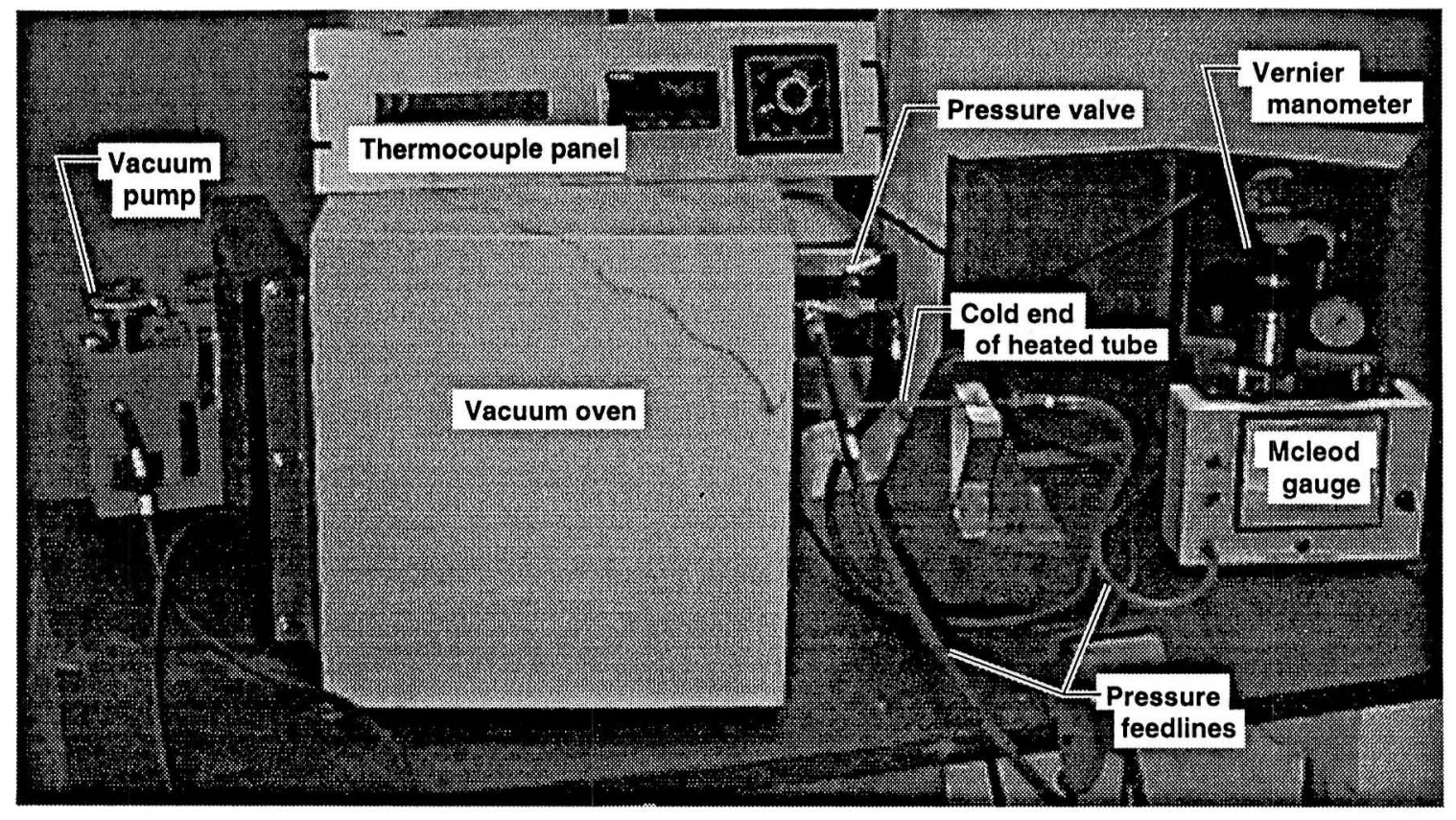

(a) Equipment layout.

Figure 4. Apparatus used for the static response and rarefied flow tests. 


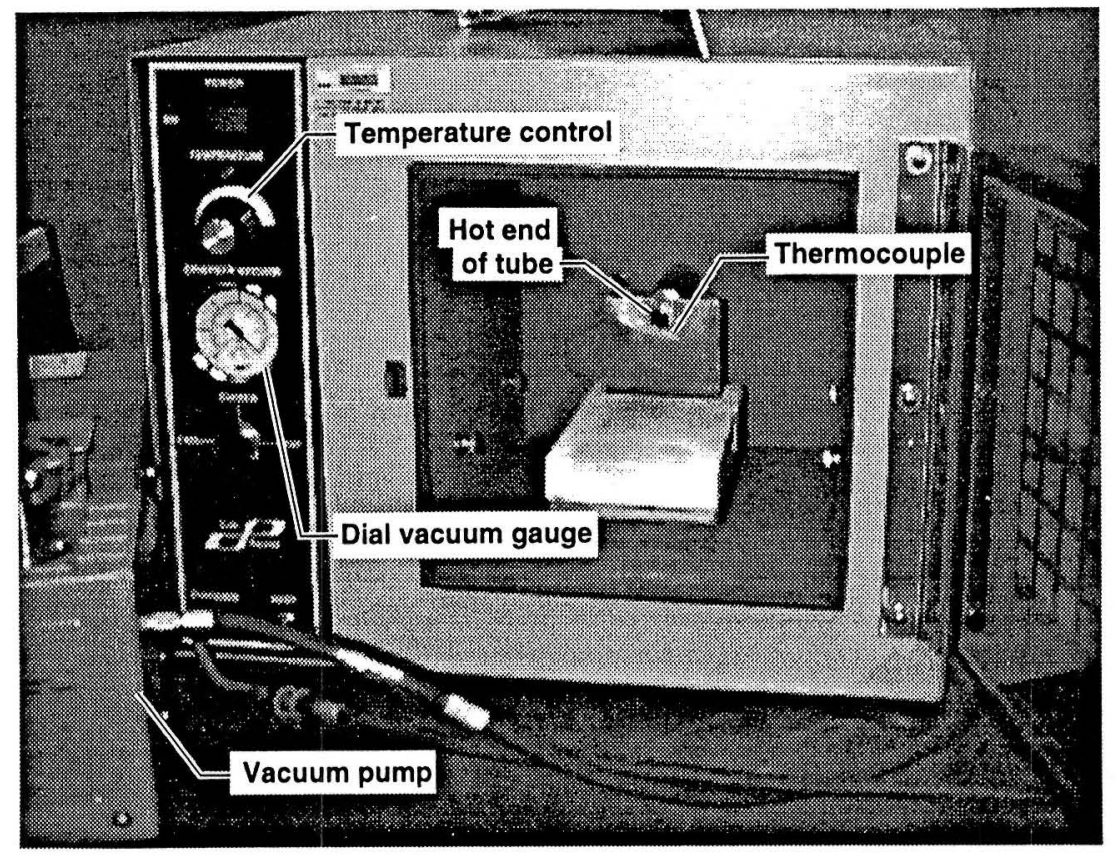

(b) Front view of vacuum oven.

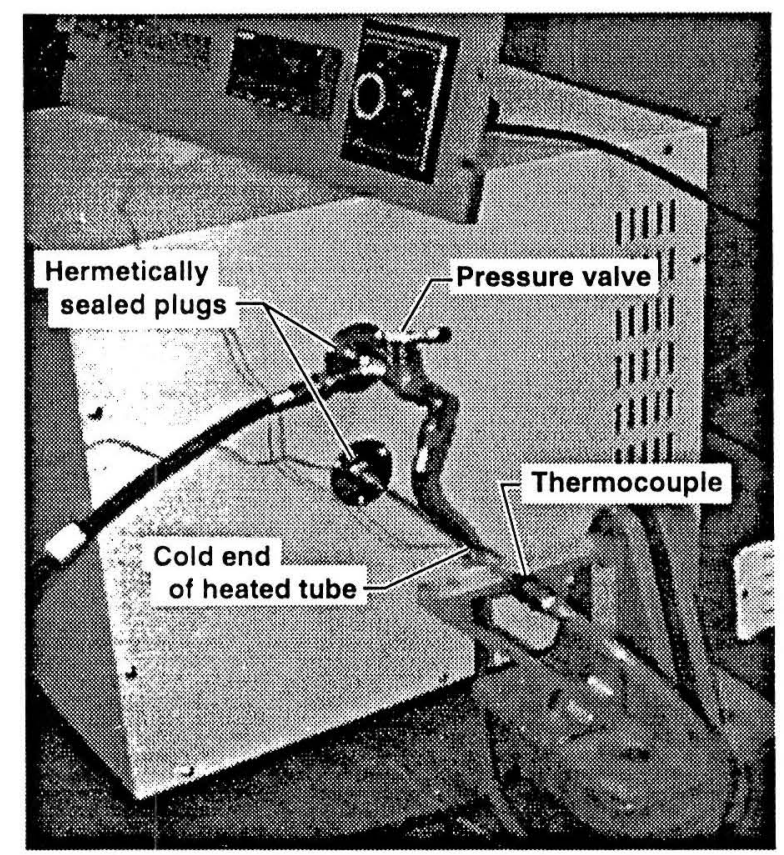

(c) Rear view showing pressure fittings.

Figure 4. Concluded. 


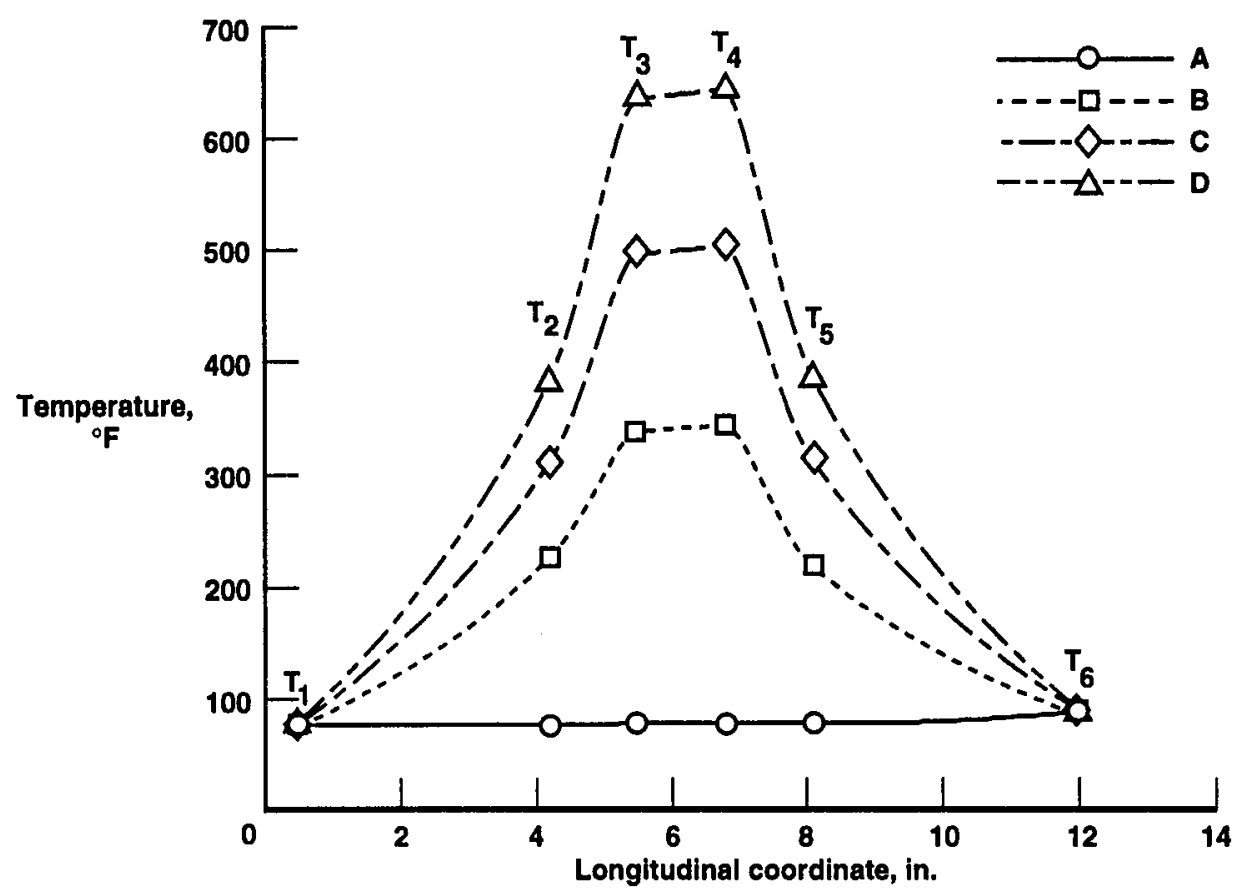

(a) Tube diameter, 0.066 in.

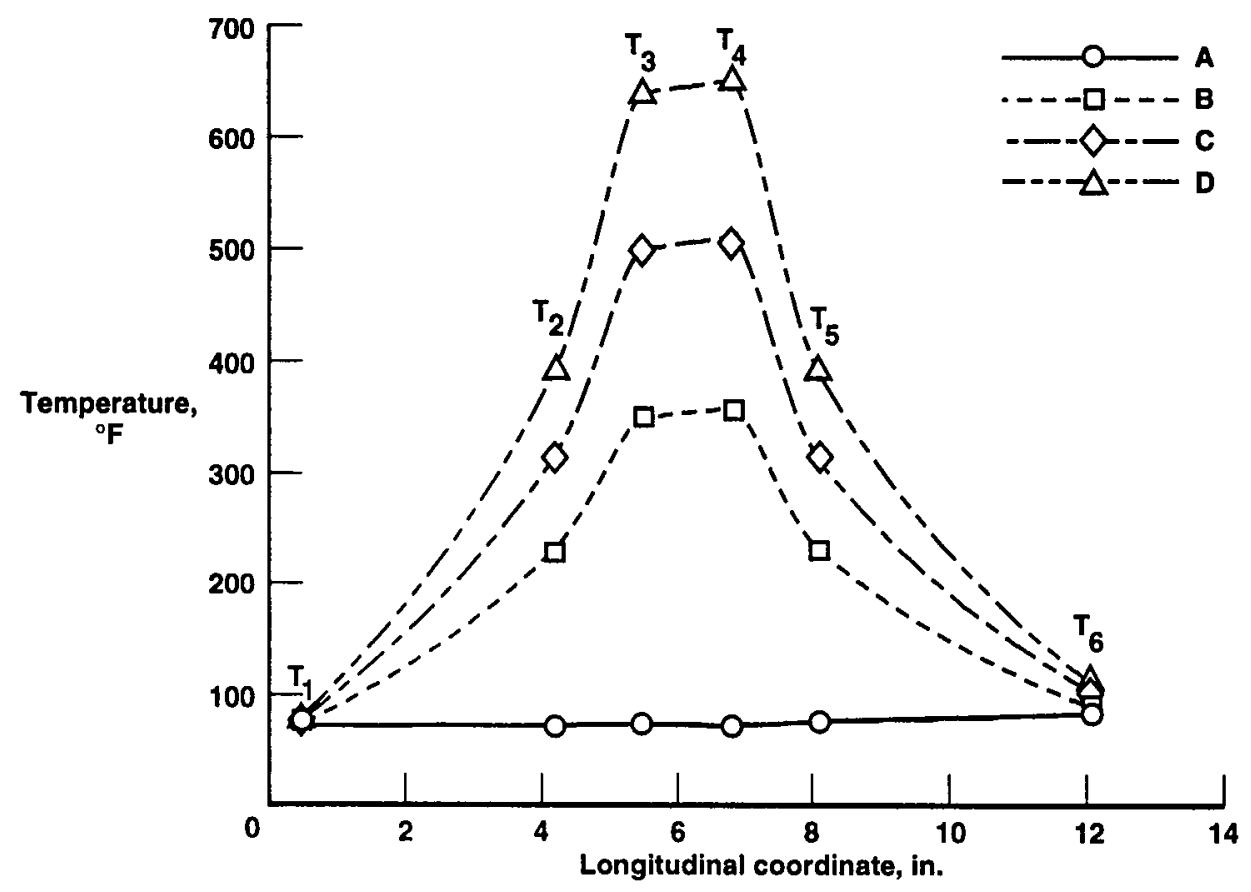

(b) Tube diameter, 0.033 in.

Figure 5. Idealized temperature profiles for frequency response tests. 

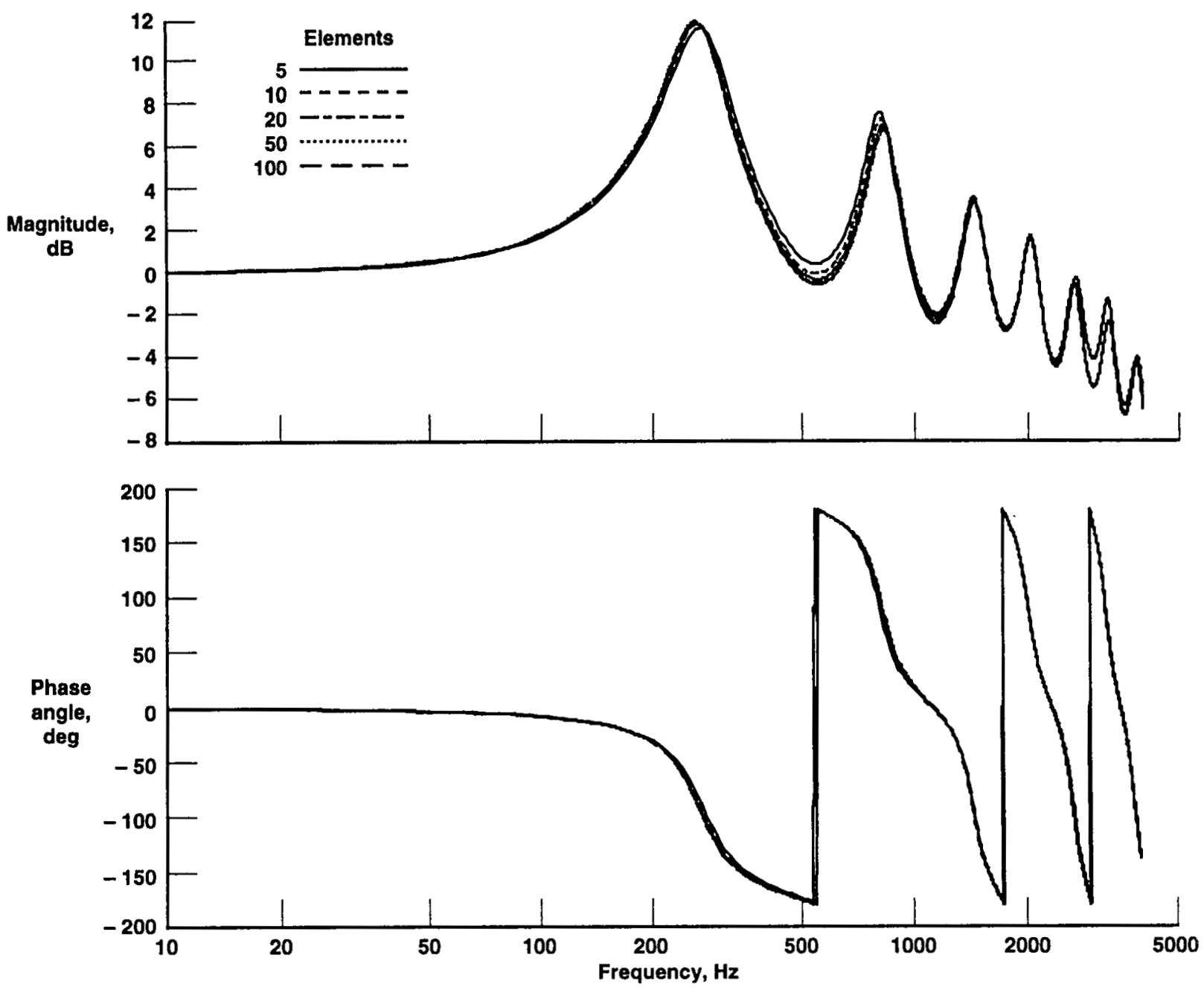

Figure 6. The effects of grid density on frequency response computation $(d=0.066 \mathrm{in}$. and temperature profile D). 

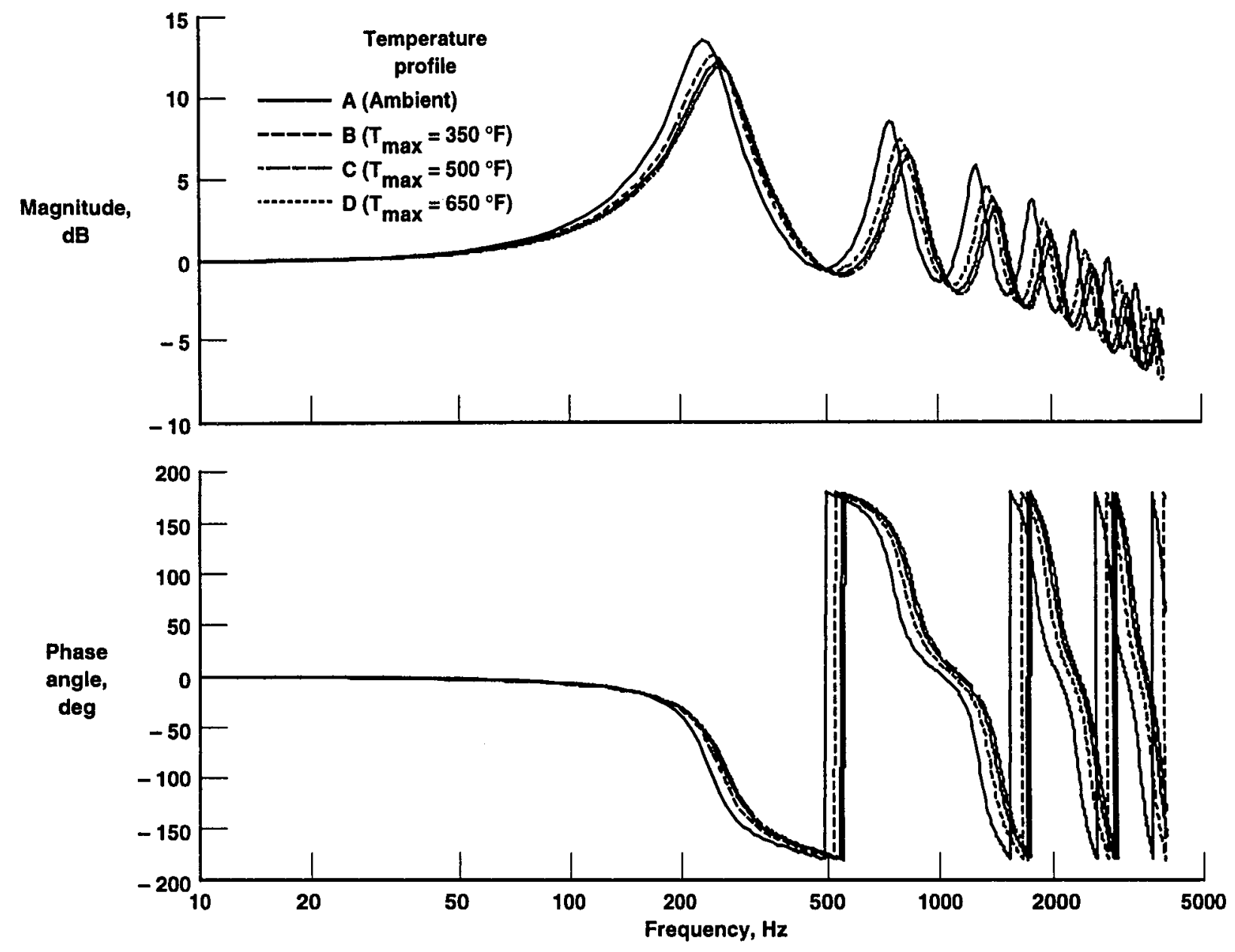

(a) $L=12$ in. and $d=0.066$-in. tube geometry.

Figure 7. Theoretical effects of temperature gradients. 

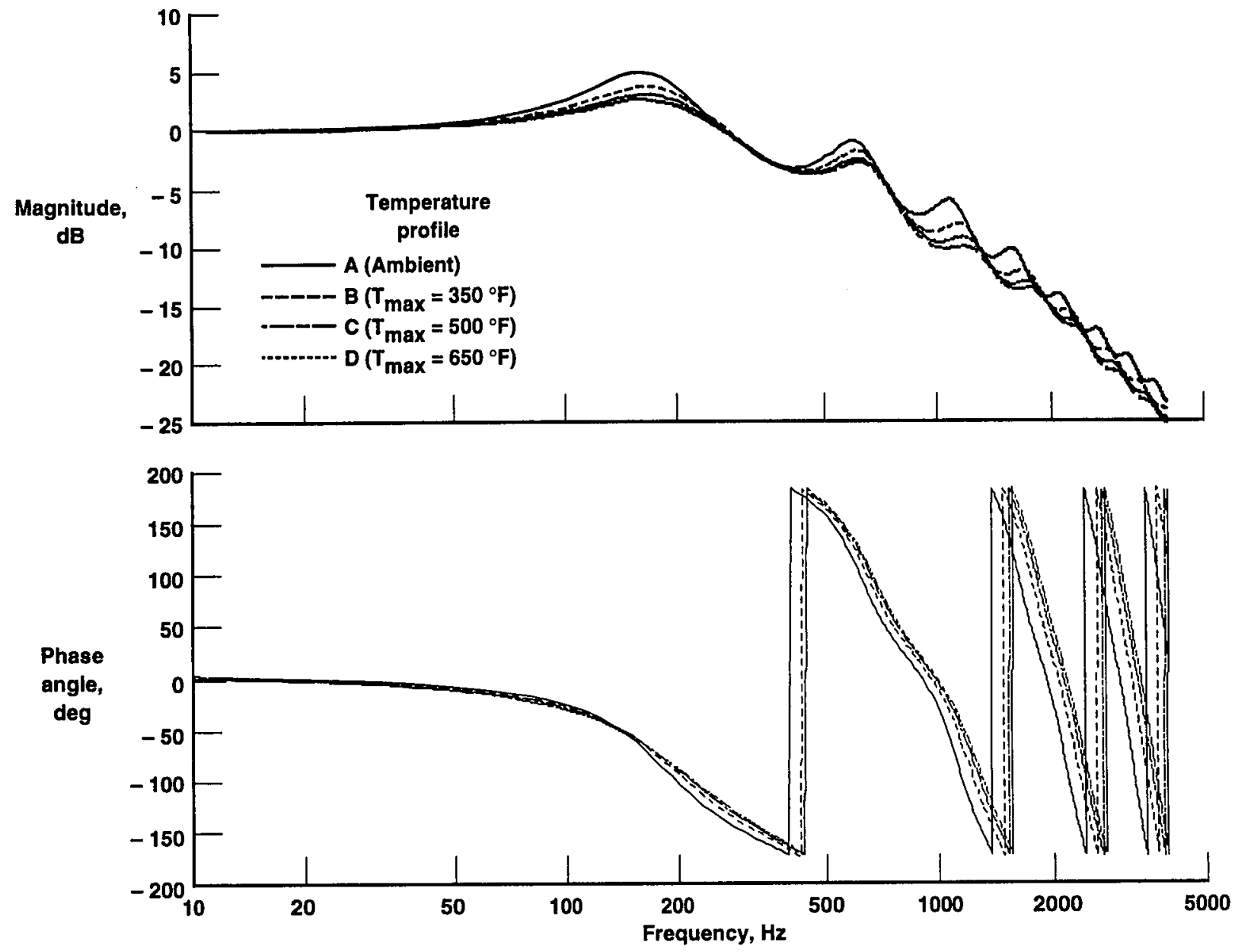

(b) $L=12$ in. and $d=0.033$-in. tube geometry.

Figure 7. Concluded. 

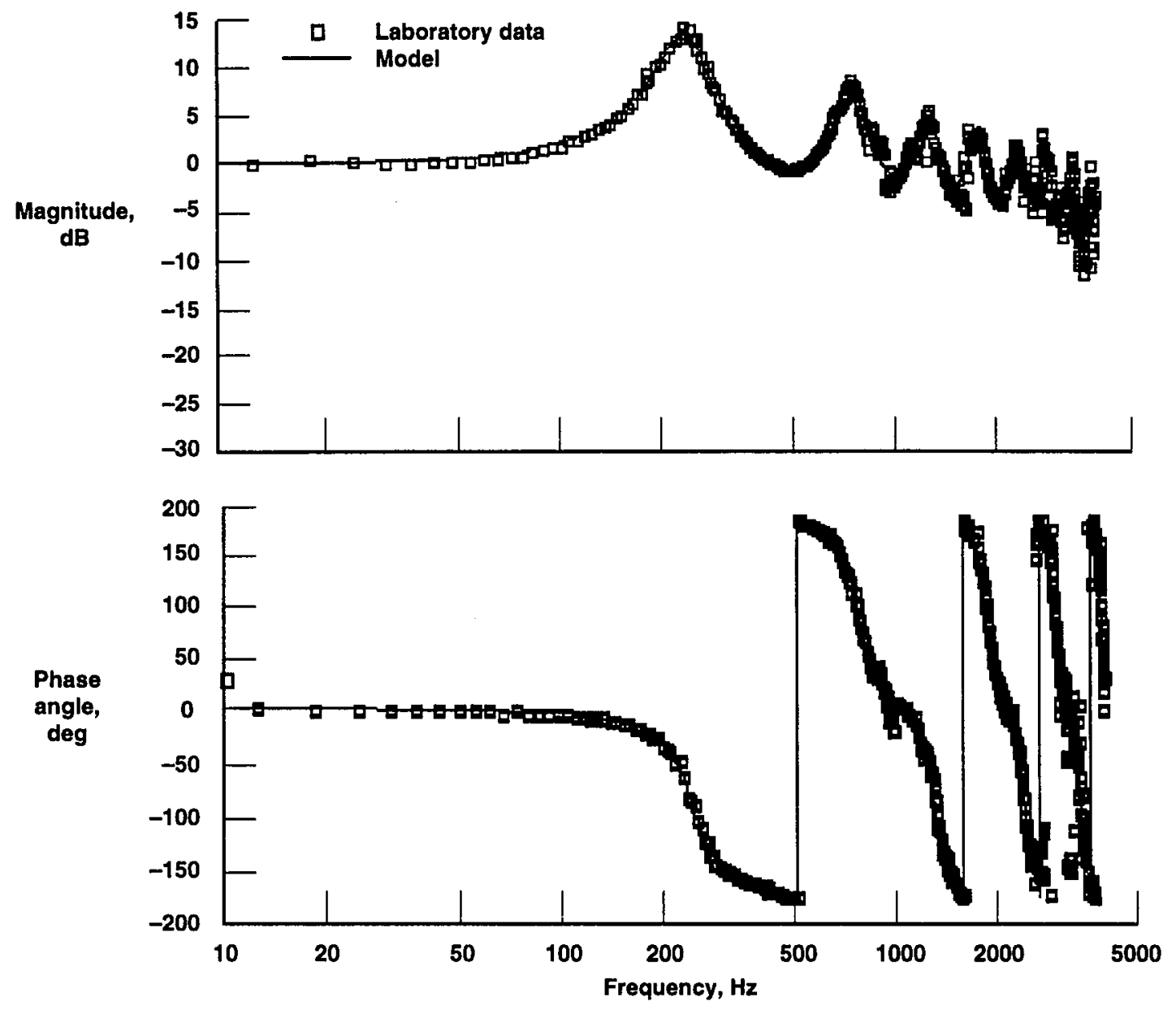

(a) Temperature profile $\mathrm{A}$ (ambient conditions).

Figure 8. Comparison of model frequency response to experimental results for $L=12$ in. and $d=0.066$-in. tubing geometry. 

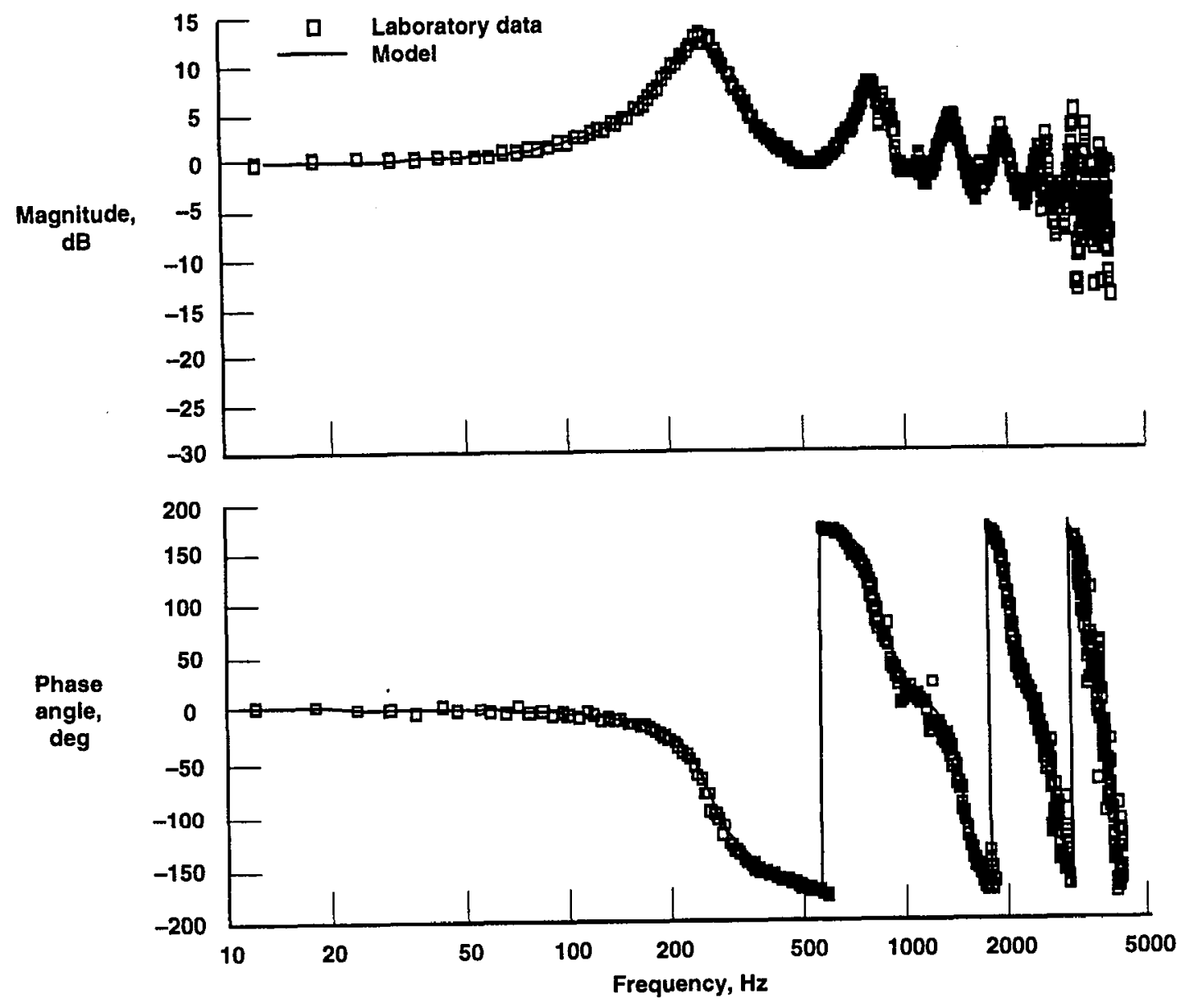

(b) Temperature profile $\mathrm{B}\left(\mathrm{T}_{\max }=350^{\circ} \mathrm{F}\right)$.

Figure 8. Continued. 

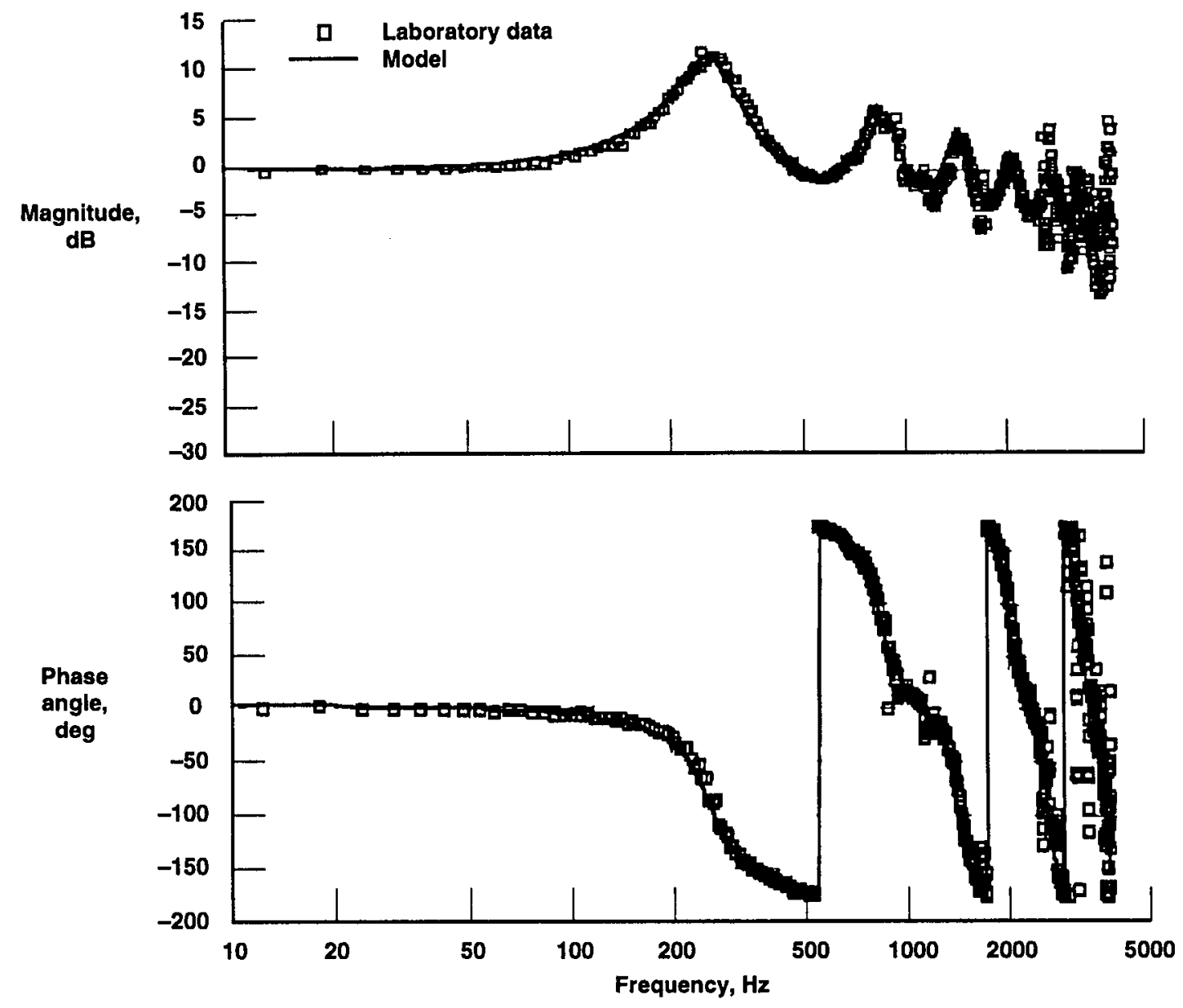

(c) Temperature profile $\mathrm{C}\left(\mathrm{T}_{\max }=500^{\circ} \mathrm{F}\right)$

Figure 8. Continued. 


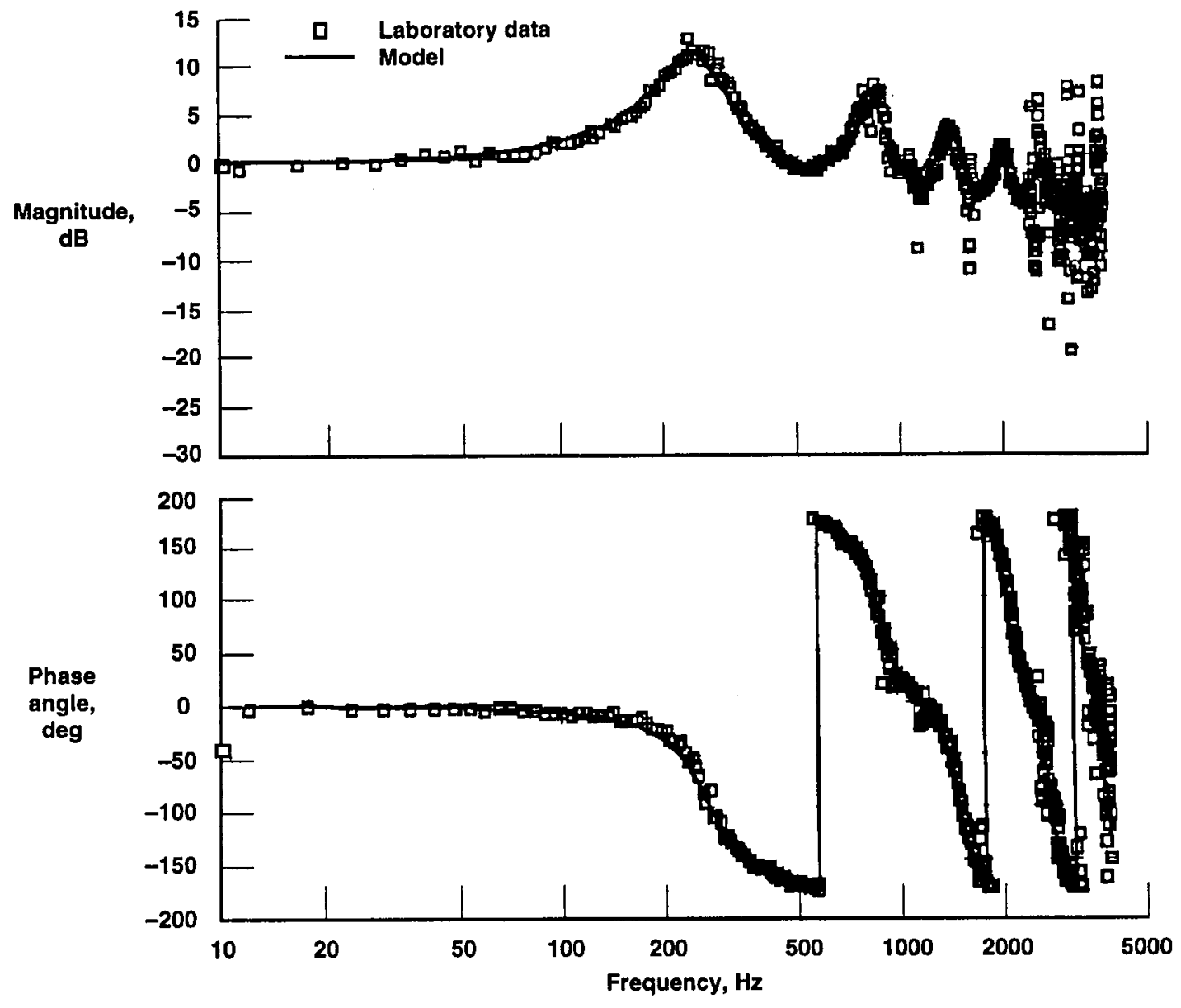

(d) Temperature profile $\mathrm{D}\left(\mathrm{T}_{\max }=650^{\circ} \mathrm{F}\right)$.

Figure 8. Concluded. 

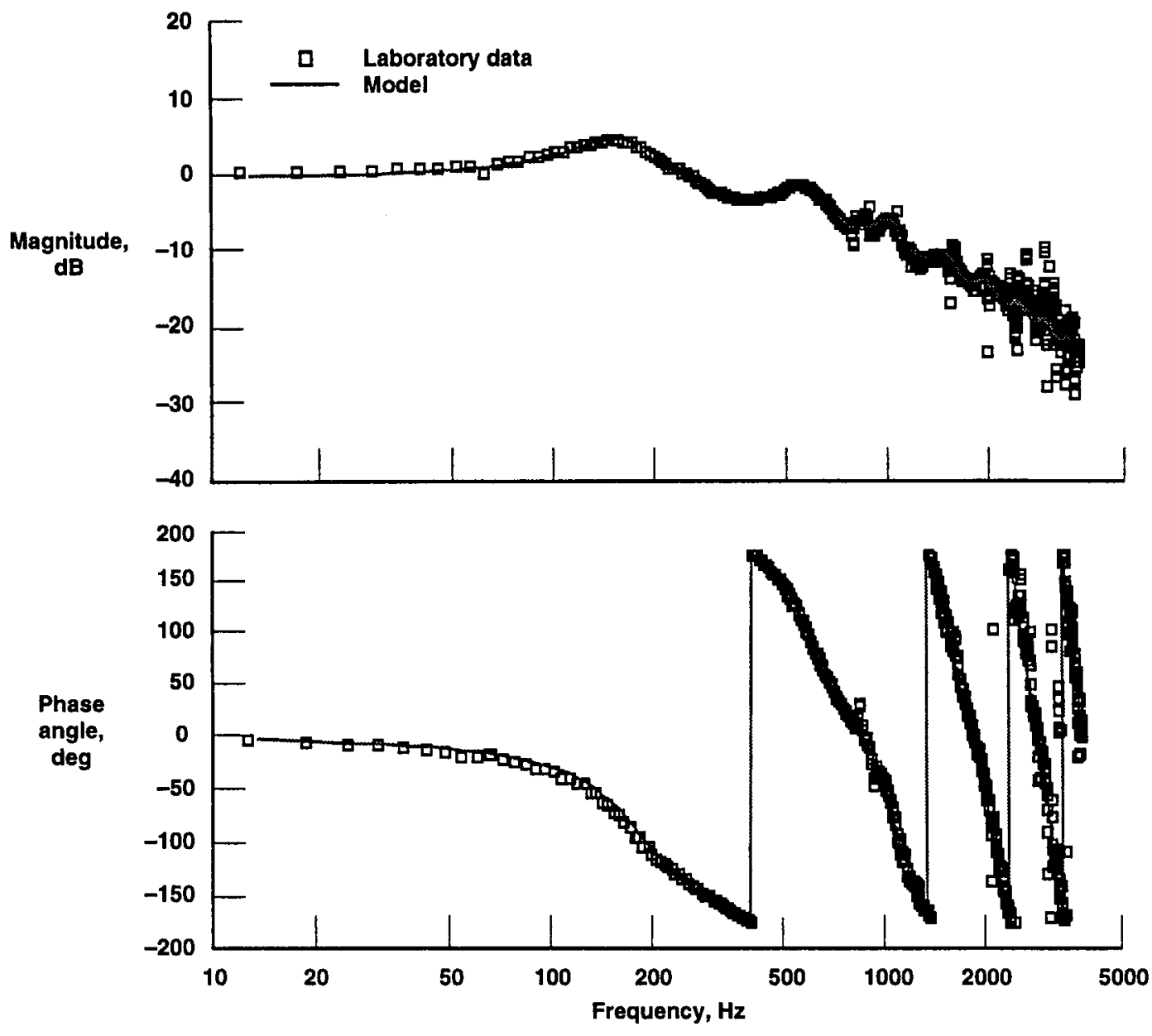

(a) Temperature profile A (ambient conditions).

Figure 9. Comparison of model frequency response to experimental results ( $L=12$ in. and $d=0.033$-in. tubing geometry). 


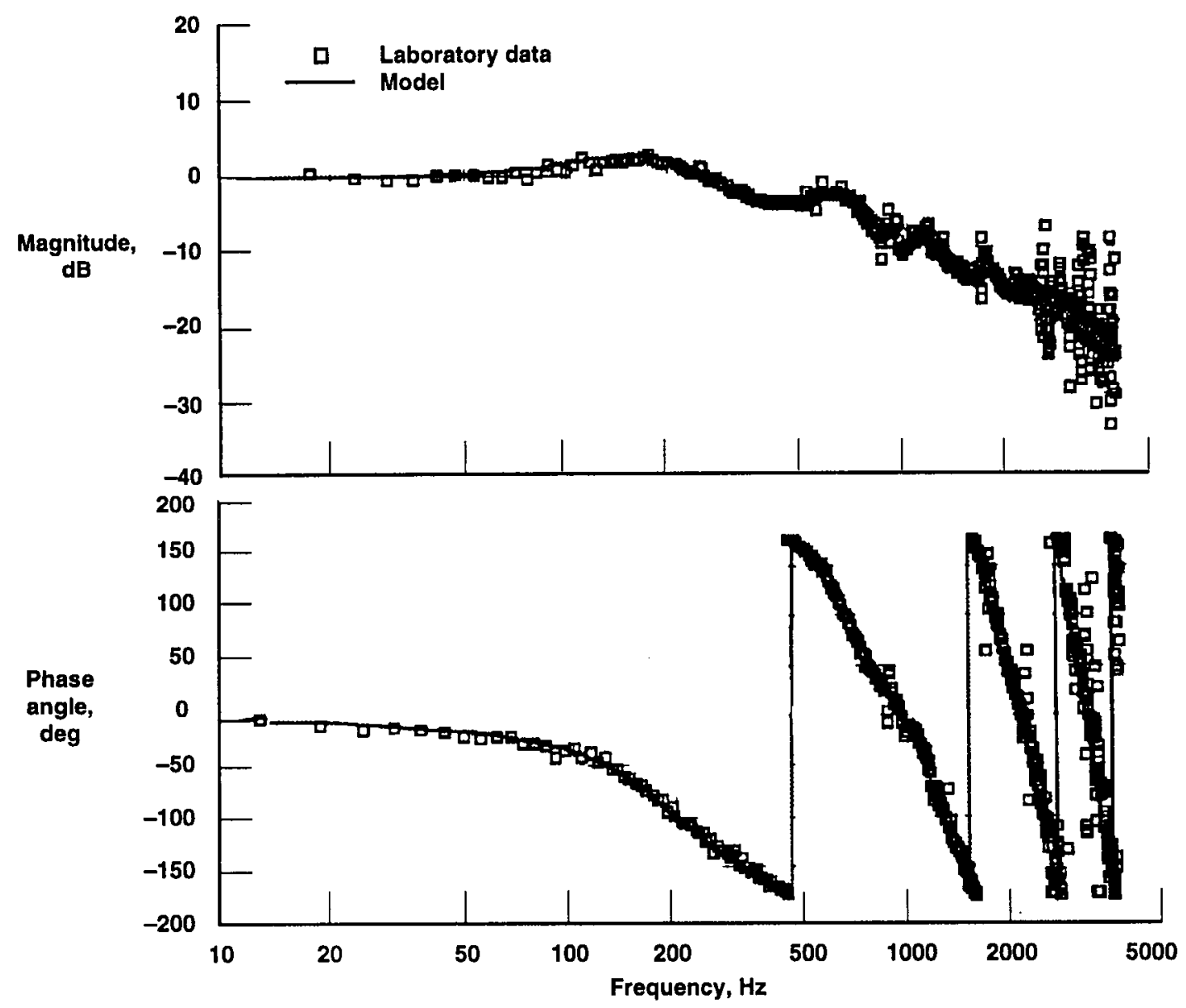

(b) Temperature profile $\mathrm{B}\left(\mathrm{T}_{\max }=350^{\circ} \mathrm{F}\right)$.

Figure 9. Continued. 

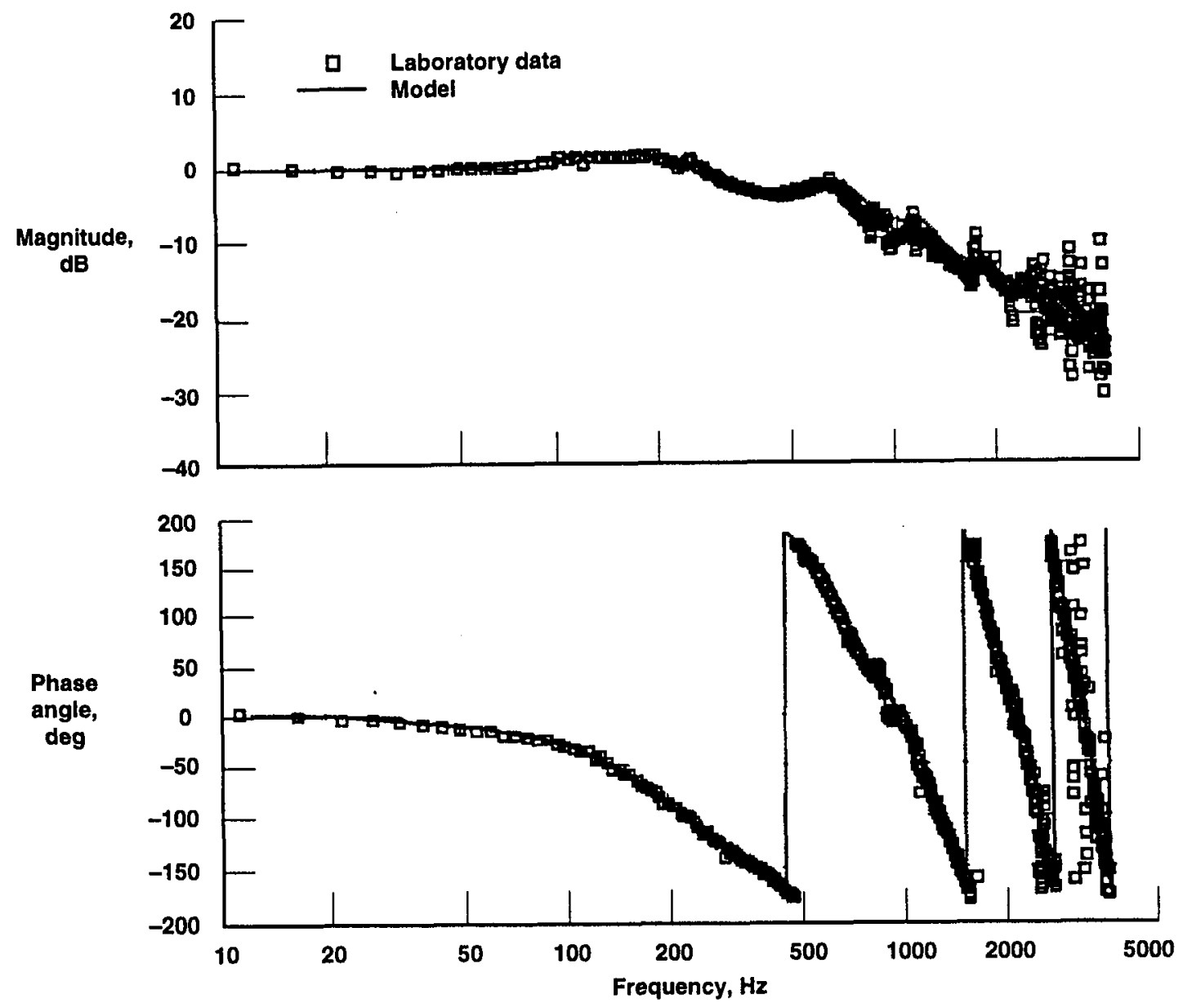

(c) Temperature profile $\mathrm{C}\left(\mathrm{T}_{\max }=500^{\circ} \mathrm{F}\right)$.

Figure 9. Continued. 

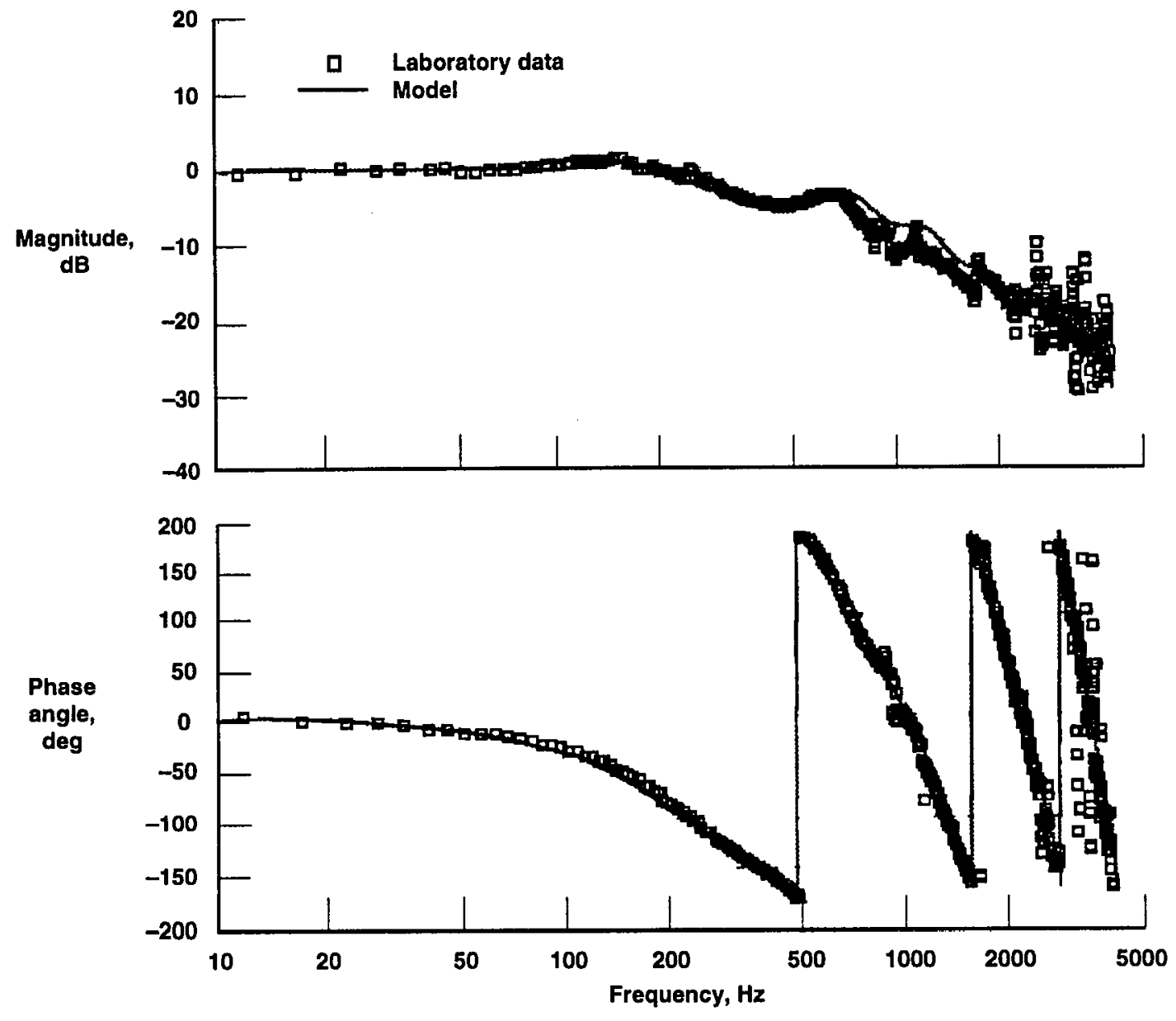

(d) Temperature profile $\mathrm{D}\left(\mathrm{T}_{\max }=650^{\circ} \mathrm{F}\right)$.

Figure 9. Concluded. 


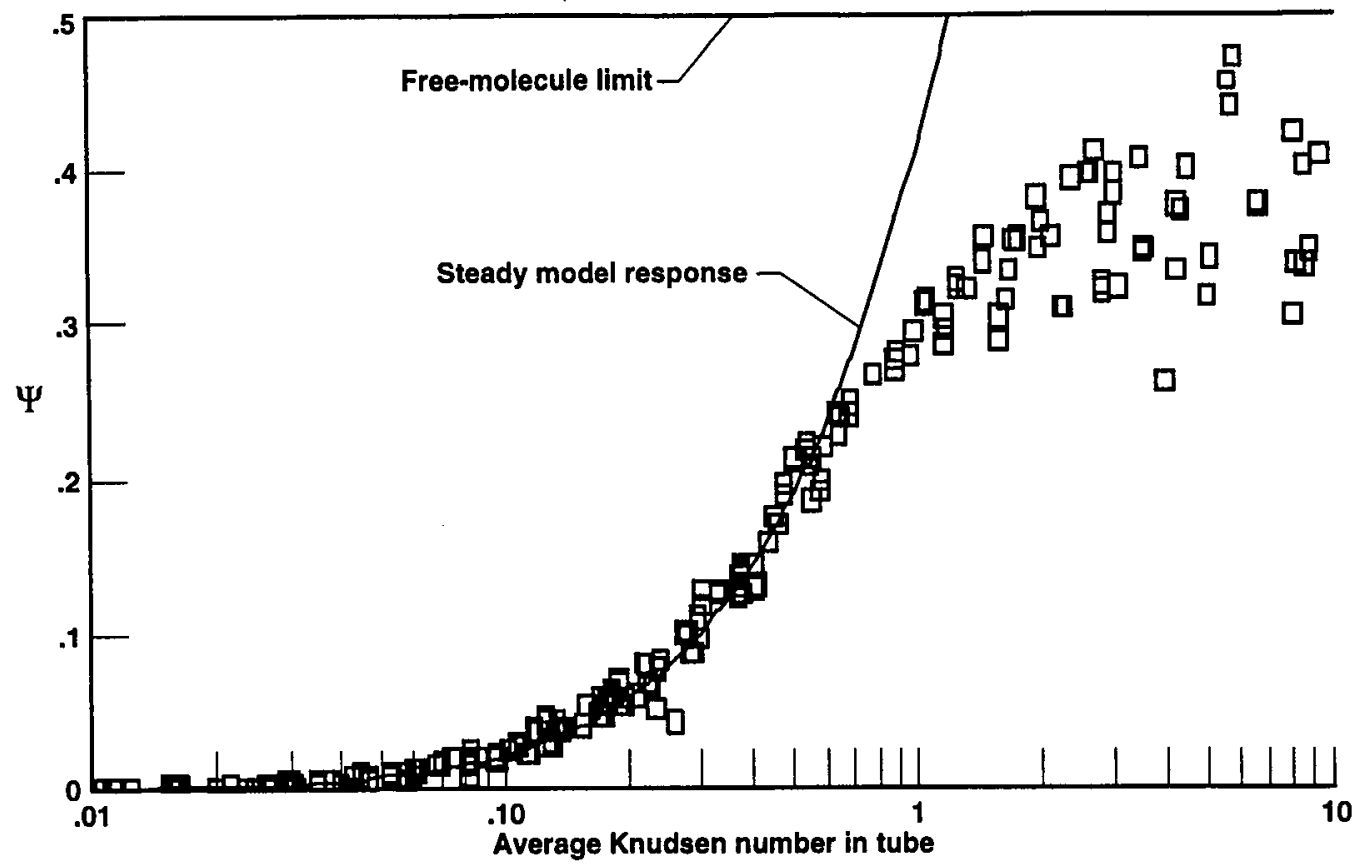

Figure 10. Comparison of steady-state model response to measured values of nondimensional steady response parameter for rarefied flow conditions.

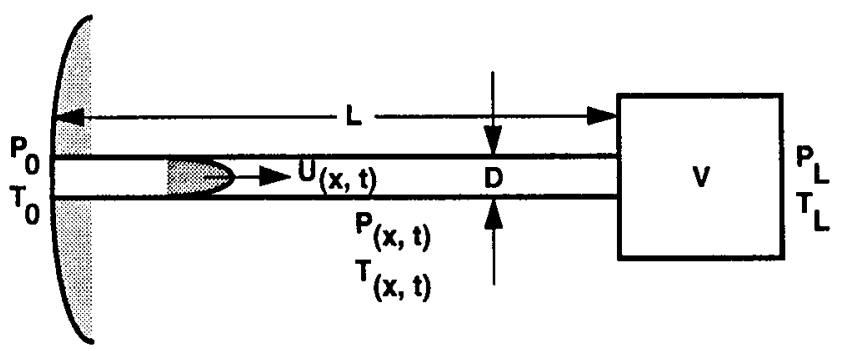

Figure A-1. Simple tubing geometry.

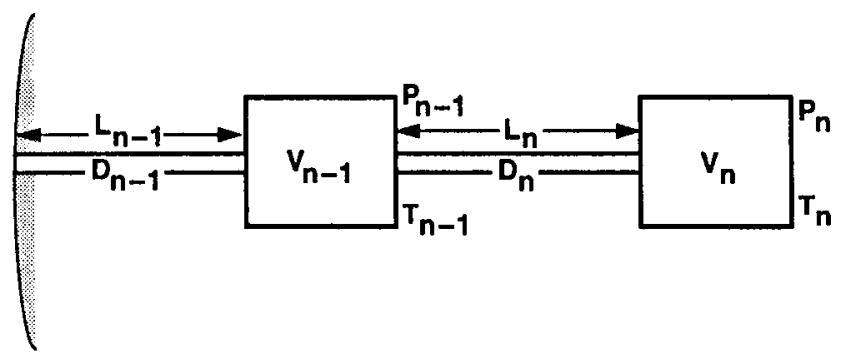

Figure A-2. Two-node geometry. 
Public reporting burden for this collection of information is estimated to average 1 hour per response, including the time for reviewing instructions, searehing existing data sources, gathering and maintaining the data needed, and completing and reviewing the collection of information. Send comments regarding this burden estimate or any other aspect of this collection of information, including suggestions for reducing this burden, to Washington Headquarters Services, Directorate for Intormation Operations and Reports, 1215 Jetferson Davis Highway, Suite 1204, Ar lington, VA 22202-4302, end to the Office of Management and Budget, Paperwork Reduction Project (0704-0188), Washington, DC 20503.

\begin{tabular}{|l|l|l}
\hline 1. AGENCY USE ONLY (Leave blank) & $\begin{array}{l}\text { 2. REPORT DATE } \\
\text { January } 1996\end{array}$ & $\begin{array}{l}\text { 3. REPORT TYPE AND DATES COVERED } \\
\text { Technical Memorandum }\end{array}$ \\
\hline
\end{tabular}

4.TITLE AND SUBTITLE

A Dynamic Response Model for Pressure Sensors in Continuum and High Knudsen Number Flows with Large Temperature Gradients

6. AUTHOR(S)

WU 52-00-RR-00-000

Stephen A. Whitmore, Brian J. Petersen, and David D. Scott

7. PERFORMING ORGANIZATION NAME(S) AND ADDRESS(ES)

NASA Dryden Flight Research Center

P.O. Box 273

Edwards, California 93523-0273

8. PERFORMING ORGANIZATION

REPORT NUMBER

H-2083

9. SPONSORING/MONOTORING AGENCY NAME(S) AND ADDRESS(ES)

10. SPONSORING/MONITORING

AGENCY REPORT NUMBER

National Aeronautics and Space Administration

Washington, DC 20546-0001

NASA TM-4728

\section{SUPPLEMENTARY NOTES}

Presented as AIAA 96-0563 at the 34th AIAA Aerospace Sciences Meeting and Exhibit, Reno, Nevada, Jan. 15-18, 1996. Stephen A. Whitmore, Dryden Flight Research Center, Edwards, California; Brian J. Petersen, UCLA, Los Angeles, California; David D. Scott, Lawrence Livermore National Laboratories, Livermore, California.

\begin{tabular}{l|l|l}
\hline 12a. DISTRIBUTION/AVAILABILITY STATEMENT & 12b. DISTRIBUTION CODE
\end{tabular}

Unclassified-Unlimited

Subject Category 02

\section{ABSTRACT (Maximum 200 words)}

This paper develops a dynamic model for pressure sensors in continuum and rarefied flows with longitudinal temperature gradients. The model was developed from the unsteady Navier-Stokes momentum, energy, and continuity equations and was linearized using small perturbations. The energy equation was decoupled from momentum and continuity assuming a polytropic flow process. Rarefied flow conditions were accounted for using a slip flow boundary condition at the tubing wall. The equations were radially averaged and solved assuming gas properties remain constant along a small tubing element. This fundamental solution was used as a building block for arbitrary geometries where fluid properties may also vary longitudinally in the tube. The problem was solved recursively starting at the transducer and working upstream in the tube. Dynamic frequency response tests were performed for continuum flow conditions in the presence of temperature gradients. These tests validated the recursive formulation of the model. Model steady-state behavior was analyzed using the final value theorem. Tests were performed for rarefied flow conditions and compared to the model steady-state response to evaluate the regime of applicability. Model comparisons were excellent for Knudsen numbers up to 0.6. Beyond this point, molecular affects caused model analyses to become inaccurate.

14. SUBJECT TERMS

Hypersonic aerodynamics; Knudsen number; Pneumatic attenuation; Pressure sensing; Rarefied flow

\begin{tabular}{|c|c|c|}
\hline $\begin{array}{c}\text { 17. SECURITY CLASSIFICATION } \\
\text { OF REPORT } \\
\text { Unclassified }\end{array}$ & $\begin{array}{c}\text { 18. SECURITY CLASSIFICATION } \\
\text { OF THIS PAGE } \\
\text { Unclassified }\end{array}$ & $\begin{array}{l}\text { 19. SECURITY CLASSIFICATION } \\
\text { OF ABSTRACT } \\
\text { Unclassified }\end{array}$ \\
\hline
\end{tabular}

NSN 7540-01-280-5500 Available from the NASA Center for AeroSpace Information, 800 Elkridge Landing Road, Linthicum Heights, MD 21090; (301)621-0390
15. NUMBER OF PAGES

43

16. PRICE CODE

$\mathrm{A} 03$

20. LIMITATION OF ABSTRACT

Unlimited 



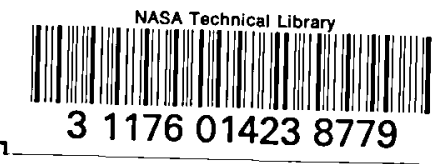

National Aeronautics and

Space Administration

Code JTT

SPECIAL FOURTH-CLASS RATE POSTAGE AND FEES PAID NASA

Washington, D.C. PERMIT No. G27

20546-0001

Official Business

Penalty for Private Use, $\$ 300$

POSTMASTER: If Undeliverable (Section 158

Postal Manual) Do Not Return 